\title{
Dynamic surface critical behavior of isotropic Heisenberg ferromagnets: boundary conditions, renormalized field theory, and computer simulation results
}

\author{
H. W. Diehl, M. Krech, , and H. Karl \\ Fachbereich Physik, Universität Essen, \\ 45117 Essen, Federal Republic of Germany
}

(Dated: November 10, 2018)

\begin{abstract}
The dynamic critical behavior of isotropic Heisenberg ferromagnets with a planar free surface is investigated by means of field-theoretic renormalization group techniques and high-precision computer simulations. An appropriate semi-infinite extension of the stochastic model J is constructed. The relevant boundary terms of the action of the associated dynamic field theory are identified, the implied boundary conditions are derived, and the renormalization of the model in $d<6$ bulk dimensions is clarified. Two distinct renormalization schemes are utilized. The first is a massless one based on minimal subtraction of dimensional poles and the dimensionality expansion about $d=6$. To overcome its problems in going below $d=4$ dimensions, a massive one for fixed dimensions $d \leq 4$ is constructed. The resulting renormalization group (or Callan Symanzik) equations are exploited to obtain the scaling forms of surface quantities like the dynamic structure factor. In conjunction with boundary operator expansions scaling relations follow that relate the critical indices of the dynamic and static infrared singularities of surface quantities to familiar static bulk and surface exponents. To test the predicted scaling forms and scaling-law expressions for the critical exponents involved, accurate computer-simulation data are presented for the dynamic surface structure factor. These are in conformity with our predictions.

PACS numbers: 75.10.Hk, 68.35.Rh, 64.60.Ht, 05.70.Jk
\end{abstract}




\section{INTRODUCTION}

A cornerstone of the modern theory of critical phenomena is the arrangement of microscopically different systems in universality classes of equivalent critical behavior. 1 . basic properties, such as the spatial dimension $d$, the order-parameter symmetry, and gross features of the interactions determine to which universality class for static bulk critical behavior a particular system belongs. These universality classes can be represented by simple continuum models like the $\phi^{4}$ model, which are minimal in the sense that dropping any of the Hamiltonian's terms implies a change of the universality class. An important alternative way of representing the universality classes is through standard lattice (spin) models such as the Ising model, which lend themselves best for precise Monte Carlo simulations.

A similar classification scheme exists for dynamic bulk critical behavior 3 The associated universality classes - called dynamic bulk universality classes henceforth — additionally depend on basic properties of the dynamics such as conservation laws, and since distinct dynamics may have the same equilibrium distribution, each static universality class generally splits up into several dynamic ones. The latter are represented by stochastic models called A, B, ... J.B

Research over the past 25 years has revealed the existence of analogous universality classes for static surface critical behavior of semi-infinite systems at bulk critical points. 슨 To which static surface universality class a given system belongs is decided by its static bulk universality class and additional relevant surface properties. Hence each static surface universality class as well as each dynamic bulk universality class usually splits up into separate dynamic surface universality classes. Furthermore, systems belonging to the same static surface universality class and the same dynamic bulk universality class may be representative of distinct dynamic surface universality classes as local changes of the dynamics at the surface can be relevant 6.8.

Unfortunately, the number of detailed theoretical investigations of dynamic surface critical behavior performed until now is rather limited.7.8.10.11.2 Furthermore, they focused more or less exclusively on models with purely relaxational dynamics. On the experimental side, the situation is worse: stringent experimental checks of the theoretical predictions for dynamic surface critical behavior, though urgently needed, are still lacking. One obvious reason for this is the difficulty of such experiments. The impressive progress made during 
the past two decades in the perfection of surface-sensitive scattering techniques has so far led only to accurate experimental investigations of static surface critical behavior. 13, 4., 45,16 Demonstrating that similarly conclusive data can also be obtained for dynamic surface critical behavior remains a major experimental challenge, albeit such experiments are expected to become feasible in the near future. According to the recent TESLA design report, 17 the X-ray free electron laser offers a great potential for such experiments.

Theoretical progress can play an important role in stimulating such experiments. We believe that theoretical advances in two directions are essential for achieving this goal. On the one hand, models representing other bulk dynamic universality classes must be considered, generalized to systems with boundaries, and carefully investigated to find out what kinds of dynamic surface critical behavior can occur, i.e., which dynamic surface universality classes exist. On the other hand, detailed theoretical predictions should be worked out for experimentally accessible quantities like structure functions etc.

Pursuing these goals, we will investigate the dynamic surface critical behavior of isotropic Heisenberg ferromagnets in this paper. Well-known characteristic features of the dynamics of such magnets are the presence of nondissipative (mode-coupling) terms and the conservation of the order parameter. We shall employ two different lines of approaches: (i) analytic work based on the field-theoretic renormalization group (RG) and (ii) computer-simulation studies of the dynamic surface structure function. A brief account of parts of our work has been given elsewhere.18

In our RG work we utilize an appropriate semi-infinite extension of the usual stochastic bulk model J, $1920202,22,23,24$ which represents the dynamic bulk universality class of the isotropic Heisenberg ferromagnet, without energy conservation. A familiar problem one is faced with is the following. Whereas the upper critical dimension of this dynamic model is $d_{\mathrm{J}}^{*}=6$, the one of its steady-state distribution, described by the usual $|\phi|^{4}$ model with an $n=3$ vector field $\phi$, is $d^{*}=4$. Thus the small parameter in which a dimensionality expansion can be made in the dynamic case is $\epsilon_{6}=6-d$ rather than $\epsilon_{4}=4-d$, where $d$ is the bulk dimension. For $4<d \leq 6$, the static critical behavior is given by mean-field theory and associated with the (then infrared-stable) Gaussian fixed point of the $|\phi|^{4}$ theory, even though the dynamic critical behavior is described by a nontrivial fixed point that is characterized by a non-zero value $f^{*}$ of the mode-coupling vertex and accessible to the $\epsilon_{6}$ expansion. 
Unfortunately, this expansion is not tailored to capture the nontrivial static critical exponents that emerge as $d$ drops below 4 . Therefore it is of somewhat limited use in the physically interesting three-dimensional case or, more generally, for $d \leq 4$. In order to to find out which scaling laws exist relating the critical exponents of dynamic bulk and surface quantities to known bulk and surface critical indices, it is essential to formulate the field-theoretic RG for fixed values of $d<4$. We do this by extending existing massive $R G$ schemes for semi-infinite system 2520 to dynamics. This yields RG (Callan-Symanzik) equations whose exploitation in conjunction with known boundary operator expansions 2728 reveals that the dynamic bulk and surface critical exponents can be expressed completely in terms of known static ones, besides giving the scaling forms of quantities like the surface structure function.

In order to check these finding we have performed high-precision computer simulations of a semi-infinite lattice model of classical Heisenberg spins whose dynamics is defined via the deterministic nondissipative equations of motion implied by their Poisson bracket relations. The advantage of this simple dynamics without noise is that recently developed extremely efficient spin dynamics algorithms 290 can be employed to compute the temporal development from given initial spin configurations, which we choose from a thermal equilibrium distribution generated via a Monte Carlo simulation.

It should be emphasized that this lattice model differs in an important aspect from the continuum model we consider: unlike the latter, it conserves the energy. Nevertheless, both models belong to the same universality class, as we intend to show.

The remainder of this paper is organized as follows. In Sec. II both the semi-infinite lattice model (studied by simulations) as well as the semi-infinite extension of the continuum model J (utilized in our RG analysis) are introduced, and their dynamics specified. The definition of the continuum model also involves the specification of appropriate boundary conditions. We discuss this question first on a heuristic basis (Sec. IIB 3). Going over to the path-integral formulation of this model in Sec. IIB4, we then show in Sec. IIB5 how the boundary conditions for both the order parameter $\phi$ and the auxiliary (Martin-Siggia-Rose) field $\tilde{\phi}$ can be justified in a systematic manner and derived from the boundary part of the dynamic action functional. Section IIB 6 briefly recalls the fluctuation-dissipation theorem and discusses the meaning of some of the boundary conditions in this context. Section 11$]$ is devoted to the RG analysis of the continuum model. After giving the free response and 
correlation propagators in Sec. IIIA, we explicate in Sec. IIIB the renormalization of the theory, describe the massless renormalization scheme on which our subsequent RG analysis in $6-\epsilon_{6}$ dimensions is based. To overcome the limitations of this scheme, we construct in Sec. [IID a massive RG scheme for fixed dimensions $d$ with $2<d \leq 4$. The resulting CallanSymanzik equations are given in Sec. [IIE and utilized to derive the scaling forms of the

correlation and response functions. Details of our Monte-Carlo spin dynamics simulation are described in Sec. IV. Its results are presented and analyzed in Sec. V. Section VI contains a brief summary and concluding remarks. Finally, in the Appendix arguments are given as to why the lattice model we study belongs to the universality class of our semi-infinite model J, even though it differs from the latter by conserving additionally energy.

\section{MODELS}

\section{A. Semi-infinite lattice Heisenberg model}

The lattice model we consider is a classical isotropic Heisenberg ferromagnet on a $d$ dimensional simple cubic lattice whose sites $\boldsymbol{i}=\left(i_{1}, \ldots, i_{d}\right)$, with $i_{\kappa}=0, \ldots, L-1$ for $\kappa=1, \ldots, d$, are occupied by spins $\boldsymbol{S}_{\boldsymbol{i}}=\left(S_{\boldsymbol{i}}^{\alpha}, \alpha=1,2,3\right)$ of length $\left|\boldsymbol{S}_{\boldsymbol{i}}\right|=1$. Free boundary conditions apply along the $i_{d}$-direction and periodic ones along the remaining $d-1$ ones, so that the layers $i_{d}=0$ and $i_{d}=L-1$ are free surfaces. The Hamiltonian of the model reads

$$
\mathcal{H}_{\text {lat }}=-J \sum_{\substack{\langle i, j\rangle \\ i_{d} \text { or } j_{d} \neq 0, L-1}} \boldsymbol{S}_{\boldsymbol{i}} \cdot \boldsymbol{S}_{\boldsymbol{j}}-J_{1} \sum_{\substack{\langle i, \boldsymbol{j}\rangle \\ i_{d}=j_{d}=0, L-1}} \boldsymbol{S}_{\boldsymbol{i}} \cdot \boldsymbol{S}_{\boldsymbol{j}}
$$

where the summations run over the specified sets of nearest-neighbor (nn) bonds $\langle\boldsymbol{i}, \boldsymbol{j}\rangle$. The bulk and surface nn interaction constants $J$ and $J_{1}$ are ferromagnetic and measured in temperature units $k_{\mathrm{B}} T$. The dynamics is defined by the equations of motion

$$
\frac{d \boldsymbol{S}_{\boldsymbol{i}}}{d t}=\frac{\partial \mathcal{H}_{\mathrm{lat}}}{\partial \boldsymbol{S}_{\boldsymbol{i}}} \times \boldsymbol{S}_{\boldsymbol{i}}
$$

which describe the precession of the spins $\boldsymbol{S}_{\boldsymbol{i}}$ in the local magnetic fields $\boldsymbol{H}_{\boldsymbol{i}} \propto-\partial \mathcal{H}_{\text {lat }} / \partial \boldsymbol{S}_{\boldsymbol{i}}$. They conserve both total spin $\sum_{i} \boldsymbol{S}_{\boldsymbol{i}}$ (in the here assumed absence of external magnetic fields) as well as total energy $E_{\text {lat }} \equiv \mathcal{H}_{\text {lat }}[\boldsymbol{S}(t)]$.

Conservation of magnetic energy is not normally considered a property of real ferromagnets since the spin system can loose energy by processes not taken into account by Eqs. (11) 
and (2) such as coupling to phonons. In fact, in the continuum model J employed in our RG analysis, only the order parameter but not the energy is conserved. Arguments as to why both models represent nevertheless the same universality class are given in Appendix $\mathrm{A}$.

In our computer simulations, a $d=3$ dimensional version of the above model is investigated. The equations of motion (21) are numerically integrated for a given set of at least 700 initial spin configurations generated by a Monte Carlo simulation of the thermal equilibrium state associated with Hamiltonian (11).29.6031 Details of this simulation are explained in Sec. [V.

Quantities of primary importance for the interpretation of scattering experiments are the spin-spin cumulant

$$
\begin{aligned}
& C^{\alpha \beta}\left(\boldsymbol{r} ; z, z^{\prime} ; t-t^{\prime}\right) \equiv\left\langle S_{\boldsymbol{i}}^{\alpha}(t) S_{\boldsymbol{i}^{\prime}}^{\beta}\left(t^{\prime}\right)\right\rangle^{\mathrm{cum}} \\
& =\left\langle S_{\boldsymbol{i}}^{\alpha}(t) S_{\boldsymbol{i}^{\prime}}^{\beta}\left(t^{\prime}\right)\right\rangle-\left\langle S_{\boldsymbol{i}}^{\alpha}(t)\right\rangle\left\langle S_{\boldsymbol{i}^{\prime}}^{\beta}\left(t^{\prime}\right)\right\rangle
\end{aligned}
$$

and its Fourier transform

$$
\begin{aligned}
& \hat{C}^{\alpha \beta}\left(\boldsymbol{p} ; z, z^{\prime} ; \omega\right) \\
& =\int d^{d-1} r e^{-i \boldsymbol{p} \cdot \boldsymbol{r}} \int_{-\infty}^{\infty} d t e^{i \omega t} C^{\alpha \beta}\left(\boldsymbol{r} ; z, z^{\prime} ; t-t^{\prime}\right) .
\end{aligned}
$$

Here $\boldsymbol{r}=\left(i_{1}-i_{1}^{\prime}, \ldots, i_{d-1}-i_{d-1}^{\prime}\right)$, while $z=i_{3}$ and $z^{\prime}=i_{3}^{\prime}$, respectively. Further, $t$ and $t^{\prime}$ are times to which the initial spin configuration at $t=0$ has evolved according to Eq. (2). The average $\langle$.$\rangle is taken over the distribution of initial configurations.$

Specifically, we will be concerned with the dynamic surface structure function

$$
\hat{C}_{11}^{\alpha \beta}(\boldsymbol{p}, \omega) \equiv \hat{C}^{\alpha \beta}(\boldsymbol{p} ; 0,0 ; \omega) .
$$

Before embarking on a discussion of its scaling properties and presenting our simulation results, it is useful to introduce first the continuum model on which our RG analysis is based.

\section{B. Semi-infinite model J}

\section{Hamiltonian of the thermal equilibrium state}

The dynamic model we are going to consider is required to satisfy detailed balance胆 32 and to ensure relaxation to a steady-state distribution corresponding to a thermal equilib- 
rium state $\propto e^{-\mathcal{H}[\phi]}$ with the Hamiltonian

$$
\mathcal{H}=\int_{\mathbb{R}_{+}^{d}}\left[\frac{1}{2}(\boldsymbol{\nabla} \boldsymbol{\phi})^{2}+\frac{\tau_{0}}{2} \phi^{2}+\frac{u_{0}}{4 !}|\boldsymbol{\phi}|^{4}\right]+\int_{\mathcal{B}} \frac{c_{0}}{2} \phi^{2} .
$$

Here the integrations extend over $\mathbb{R}_{+}^{d} \equiv\left\{\left(\boldsymbol{x}_{\|}, z\right) \in \mathbb{R}^{d} \mid z \geq 0\right\}$, the $d$-dimensional half-space, and $\mathcal{B}$, its $(d-1)$-dimensional boundary plane at $z=0$, respectively. The order-parameter density $\boldsymbol{\phi}=\left(\phi^{\alpha}\right)$ is a three-vector.

Above $d=3$ bulk dimensions, this static model is known to undergo at the bulk critical point so-called ordinary, special, and extraordinary surface transitions, depending on whether the surface enhancement variable $c_{0}$ is larger than, equal to, or less than a critical value $c_{\mathrm{sp}}$ 晛 For $d=3$, the surface cannot spontaneously order at the bulk critical temperature $T_{\mathrm{c}}>0$ because of the presumed continuous $O(3)$ symmetry of the Hamiltonian (6). Hence only the ordinary transition remains in this case. Analogous statements apply to the lattice model (11), for whose $d>3$ variant the role of the variable $-c_{0}$ is played by the 'surface enhancement' $\left(J_{1} / J\right)-\left(J_{1} / J\right)_{\mathrm{sp}}$, where $\left(J_{1} / J\right)_{\mathrm{sp}}$ is the critical value of the ratio $J_{1} / J$ pertaining to the special transition.

\section{Langevin equations}

Next we turn to the task of formulating an appropriate semi-infinite extension of the standard bulk model J. For reasons expounded elsewhere, , $^{\mathrm{B}}$ we may assume that the surfaceinduced modifications of both the interactions as well as the dynamics are restricted to the immediate vicinity of the boundary $\mathcal{B}$. Consequently, we use the stochastic bulk equation

$$
\dot{\boldsymbol{\phi}}(\boldsymbol{x}, t)=\lambda_{0}\left(\triangle \mathcal{H}_{\boldsymbol{\phi}}+f_{0} \mathcal{H}_{\boldsymbol{\phi}} \times \boldsymbol{\phi}\right)+\boldsymbol{\zeta}(\boldsymbol{x}, t)
$$

for all points $\boldsymbol{x}$ with $z>0$. Here $\boldsymbol{\zeta}$ is a Gaussian random force with average $\langle\boldsymbol{\zeta}\rangle=0$ and variance

$$
\left\langle\zeta^{\alpha}(\boldsymbol{x}, t) \zeta^{\beta}\left(\boldsymbol{x}^{\prime}, t^{\prime}\right)\right\rangle=-2 \lambda_{0} \delta^{\alpha \beta} \triangle \delta\left(\boldsymbol{x}-\boldsymbol{x}^{\prime}\right) \delta\left(t-t^{\prime}\right) .
$$

Further, $\mathcal{H}_{\phi}$ stands for the part of the functional derivative

$$
\frac{\delta \mathcal{H}}{\delta \phi}(\boldsymbol{x}, t)=\mathcal{H}_{\phi}(\boldsymbol{x}, t)+\delta(z)\left(c_{0}-\partial_{n}\right) \boldsymbol{\phi}(\boldsymbol{x}, t)
$$


that remains away from the boundary plane $\mathcal{B}$, namely

$$
\mathcal{H}_{\phi}(\boldsymbol{x}, t)=\left(-\triangle+\tau_{0}+\frac{u_{0}}{6}|\phi|^{2}\right) \boldsymbol{\phi} .
$$

The derivative $\partial_{n}$ in Eq. (9) is along the inner normal, i.e., $\partial_{n}=\partial_{z}$ on $\mathcal{B}$.

In order to extend the model to the semi-infinite case, we must specify whether and how Eqs. (7) and (8) are to be modified in the vicinity of $\mathcal{B}$. Owing to our locality assumption mentioned at the beginning of this sub-subsection, this should amount to a choice of boundary conditions for $\phi$. For the sake of simplicity, we assume that the conservation of the order parameter is not violated by boundary contributions. This is physically reasonable since we took all bulk and surface terms of the Hamiltonian (6) to have $O(3)$ symmetry, as is appropriate for a Heisenberg magnet whose interactions are isotropic even at the surface.

\section{Boundary conditions for $\phi$}

Building on previous work on model B 89.9 , we can now easily anticipate the proper boundary conditions. One boundary condition for $\phi$ should be the usual static one

$$
\partial_{n} \phi=c_{0} \phi
$$

which ensures the vanishing of the contribution $\propto \delta(z)$ of the functional derivative (9).

The other one is entailed by the required order-parameter conservation. This becomes clear if we rewrite Eq. (7) as a continuity equation:

$$
\dot{\phi}^{\alpha}+\nabla \cdot\left(\boldsymbol{j}^{(\alpha)}+\boldsymbol{j}_{\zeta}^{(\alpha)}\right)=0
$$

Here

$$
\boldsymbol{j}^{(\alpha)}=-\lambda_{0}\left(\nabla \mathcal{H}_{\phi^{\alpha}}+f_{0} \epsilon^{\alpha \beta \gamma} \phi^{\beta} \nabla \phi^{\gamma}\right)
$$

are the deterministic parts of the currents, and the noise parts satisfy

$$
\zeta^{\alpha}=-\nabla \cdot j_{\zeta}^{(\alpha)}
$$

To ensure conservation of the total order parameter, no currents must leave the system. Hence the normal component of the currents should vanish,

$$
j_{n}^{(\alpha)} \equiv \boldsymbol{n} \cdot \boldsymbol{j}^{(\alpha)}=0, \quad \alpha=1,2,3
$$


If spin anisotropies were present at the surface (which is not uncommon), the conservation would be violated at the surface for some, if not all, components of $\phi$.

Both boundary conditions, Eqs. (11) and (15), are valid in an operator sense, i.e., hold inside of averages over the initial values and the noise (yielding correlation and response functions). Note that the validity of the former has two immediate consequences: The surface contributions to the currents one would expect from the $\delta$-function term of Eq. (9) upon using the definition $\boldsymbol{j}^{(\alpha)}=-\lambda_{0} \boldsymbol{\nabla} \delta \mathcal{H} / \delta \phi^{\alpha}$ rather than Eq. (13) disappears. Furthermore, substitution of the boundary condition (11) into Eq. (15) shows that the precession term's contributions $\left(\propto f_{0}\right)$ to the currents $\boldsymbol{j}^{(\alpha)}, \alpha=1,2,3$, vanish, so that these latter boundary conditions become

$$
\partial_{n} \mathcal{H}_{\phi} \equiv \partial_{n}\left(-\triangle+\tau_{0}+\frac{u_{0}}{6}|\phi|^{2}\right) \phi=0
$$

The probability distribution of the noise clearly must also comply with the presumed order-parameter conservation. We prefer to discuss the consequences within the framework of the functional-integral (re-)formulation of the theory, 21,22.32.33 where they manifest themselves as boundary conditions for the auxiliary or Martin-Siggia-Rose 34 (MSR) field $\tilde{\phi}$ introduced below.

\section{Functional-integral formulation}

The Langevin equations (『) can be rewritten as

$$
\dot{\phi}(\boldsymbol{x}, t)=-\left(\boldsymbol{\mathcal { R }} \cdot \frac{\delta \mathcal{H}}{\delta \phi}\right)(\boldsymbol{x}, t)+\boldsymbol{\zeta}(\boldsymbol{x}, t)
$$

where $\mathcal{R}=\left(\mathcal{R}^{\alpha \beta}\right)$ denotes the reaction operator

$$
\mathcal{R}^{\alpha \beta}=-\lambda_{0}\left(\delta^{\alpha \beta} \triangle+f_{0} \epsilon^{\alpha \beta \gamma} \phi^{\gamma}\right)
$$

Since this operator acts on $\mathcal{H}_{\phi}$, which according to Eq. (16) satisfies a Neumann boundary condition, the Laplacian it involves is self-adjoint on an appropriate space of (sufficiently smooth) functions satisfying this boundary condition.

The measure $e^{-\mathcal{J}[\tilde{\boldsymbol{\phi}}, \boldsymbol{\phi}]} \mathcal{D}[\tilde{\boldsymbol{\phi}}, \boldsymbol{\phi}]$ which appears in the equivalent functional-integral formulation21,22,32,33 of the theory can now easily be inferred. To this end, let us first recall

which form the action $\mathcal{J}[\tilde{\phi}, \phi]$ must have to ensure detailed balance and relaxation to the 
chosen equilibrium state. For the here considered case in which the noise has a Gaussian probability distribution, this is 21.32

$$
\mathcal{J}=\int_{-\infty}^{\infty} d t \int_{\boldsymbol{x}}\left\{\tilde{\boldsymbol{\phi}} \cdot\left[\dot{\phi}+\overleftrightarrow{\mathcal{R}} \cdot\left(\frac{\delta \mathcal{H}}{\delta \phi}-\tilde{\phi}\right)-\frac{\delta \overleftrightarrow{\mathcal{R}}}{\delta \phi}\right]\right\}
$$

where a pre-point discretization of time is understood to be employed.

The action of the bulk model $\mathrm{J}$ is known to be of this form, with the reaction operator $\overleftrightarrow{\mathcal{R}}$ being given by Eq. (18). If we accept the boundary conditions (11) and (16), then contributions to the action that are localized on the surface vanish. Consequently this result for the action must also hold in the semi-infinite case we considered, with $\int_{\boldsymbol{x}}$ interpreted as the volume integral $\int_{\mathbb{R}_{+}^{d}}$.

Conversely, one can start from an action of form (19) and derive the boundary conditions in a systematic fashion 35 along the lines followed in Refs. 0 and 8 . The various general assumptions we have made (consistency with bulk model $\mathrm{J}$, only local modifications of the dynamics at the surface, absence of nonconservative surface terms, etc.) can be combined with relevance/irrelevance considerations to conclude that the reaction operator reads

$$
\overleftrightarrow{\mathcal{R}}=\lambda_{0}\left(\delta^{\alpha \beta} \overleftarrow{\nabla} \vec{\nabla}-f_{0} \epsilon^{\alpha \beta \gamma} \phi^{\gamma}\right)
$$

where $\overleftarrow{\nabla}$ acts to the left. This is identical to Eq. (18) up to the replacement of the Laplacian by the symmetric expression $\overleftarrow{\nabla} \vec{\nabla}$

The substitution of this form of $\overleftrightarrow{\mathcal{R}}$ into Eq. (19) and an integration by parts (making no use of the boundary conditions (11) and (16)) yields

$$
\begin{aligned}
\mathcal{J}= & \int_{-\infty}^{\infty} d t\left(\int _ { \mathbb { R } _ { + } ^ { d } } \left\{\tilde{\boldsymbol{\phi}} \cdot\left(\dot{\boldsymbol{\phi}}-\lambda_{0} f_{0} \mathcal{H}_{\boldsymbol{\phi}} \times \boldsymbol{\phi}\right)\right.\right. \\
& \left.-\lambda_{0}\left(\mathcal{H}_{\boldsymbol{\phi}}-\tilde{\boldsymbol{\phi}}\right) \triangle \tilde{\boldsymbol{\phi}}\right\} \\
& -\lambda_{0} \int_{\mathcal{B}}\left\{(\triangle \tilde{\boldsymbol{\phi}}) \cdot\left(c_{0}-\partial_{n}\right) \boldsymbol{\phi}+\left[\mathcal{H}_{\boldsymbol{\phi}}-\tilde{\boldsymbol{\phi}}\right.\right. \\
& \left.\left.\left.+\delta(z)\left(c_{0}-\partial_{n}\right) \boldsymbol{\phi}-f_{0} \boldsymbol{\phi} \times \tilde{\boldsymbol{\phi}}\right] \cdot \partial_{n} \tilde{\boldsymbol{\phi}}\right\}\right) .
\end{aligned}
$$

The singular piece $\propto \delta(z=0)$ present in the boundary integral $\int_{\mathcal{B}}$ is familiar from Refs. 7,8, 9 . It results from the coincidence of two $\delta$ functions. This singularity can be cured by replacing one of the $\delta$ functions by a smeared-out smooth analog such as $\delta_{B}(z) \equiv B e^{-B z}$, with a large positive but finite value of $B$. 


\section{Boundary conditions as boundary equations of motion}

Starting from the action (19), we can now obtain the boundary conditions for both $\phi$ and $\tilde{\phi}$ in a standard manner胭 as 'boundary contributions to the equations of motion'. This works as follows. We add source terms $\mathcal{J}_{j}[\tilde{\boldsymbol{\phi}}, \boldsymbol{\phi}]$ to the action and consider the generating functional $\mathcal{Z}[j] \equiv \int \mathcal{D}[\tilde{\boldsymbol{\phi}}, \boldsymbol{\phi}] e^{-\mathcal{J}-\mathcal{J}_{j}}$, where $j$ stands for the set of all sources considered, including eventual ones localized on the surface. That is, the source part of the action can be written as

$$
\mathcal{J}_{j}[\tilde{\boldsymbol{\phi}}, \boldsymbol{\phi}]=-\int_{-\infty}^{\infty} d t\left[\int_{\mathbb{R}_{+}^{d}} j_{\kappa} O_{\kappa}+\int_{\mathcal{B}} j_{\rho} O_{\rho}\right]
$$

where $O_{\kappa}=O_{\kappa}(\boldsymbol{x}, t)$ and $O_{\rho}=O_{\rho}\left(\boldsymbol{x}_{\|}, t\right)$ are local functionals of $\boldsymbol{\phi}$ and $\tilde{\boldsymbol{\phi}}$. From the invariance of the generating functional $\mathcal{Z}[j]$ with respect to a change of variables $\phi \rightarrow \phi+\delta \phi$ and $\tilde{\phi} \rightarrow \tilde{\phi}+\delta \tilde{\phi}$ with arbitrary (smooth) functions $\delta \phi$ and $\delta \tilde{\phi}$ we may then conclude that

$$
\left\langle\delta \mathcal{J}+\delta \mathcal{J}_{j}\right\rangle_{j}=0
$$

Here $\delta \mathcal{J}$ and $\delta \mathcal{J}_{j}$ are the implied changes of first order in $\delta \phi$ and $\delta \tilde{\phi}$ of the action $\mathcal{J}$ and its source part $\mathcal{J}_{j}$, respectively, and $\langle.\rangle_{j}$ denotes the average in the presence of sources.

Explicitly, we have

$$
\begin{aligned}
\delta \mathcal{J}= & \int_{-\infty}^{\infty}\left\{\int_{\mathbb{R}_{+}^{d}}\left[\mathcal{J}_{\phi} \delta \phi+\mathcal{J}_{\tilde{\phi}} \delta \tilde{\phi}\right]\right. \\
& +\int_{\mathcal{B}}\left[\mathcal{J}_{\phi_{\mathcal{B}}} \delta \phi+\mathcal{J}_{\tilde{\phi}_{\mathcal{B}}} \delta \tilde{\phi}\right. \\
& +\mathcal{J}_{\partial_{n} \phi} \partial_{n} \delta \phi+\mathcal{J}_{\partial_{n} \tilde{\phi}} \partial_{n} \delta \tilde{\phi} \\
& \left.\left.+\mathcal{J}_{(\triangle \phi)_{\mathcal{B}}} \triangle \delta \phi+\mathcal{J}_{(\triangle \tilde{\phi})_{\mathcal{B}}} \triangle \delta \tilde{\phi}\right]\right\}
\end{aligned}
$$

with

$$
\begin{gathered}
\mathcal{J}_{\phi}=-\partial_{t} \tilde{\boldsymbol{\phi}}-\lambda_{0}\left[-\triangle+\boldsymbol{U}_{2}\right] \triangle \tilde{\boldsymbol{\phi}} \\
-\lambda_{0} f_{0}\left[\triangle(\tilde{\boldsymbol{\phi}} \times \boldsymbol{\phi})+\tilde{\boldsymbol{\phi}} \times \mathcal{H}_{\phi}\right], \\
\mathcal{J}_{\tilde{\boldsymbol{\phi}}}=\partial_{t} \boldsymbol{\phi} \\
-\lambda_{0} \triangle\left(\mathcal{H}_{\boldsymbol{\phi}}-2 \tilde{\boldsymbol{\phi}}\right)-\lambda_{0} f_{0} \mathcal{H}_{\boldsymbol{\phi}} \times \boldsymbol{\phi}, \\
\mathcal{J}_{\phi_{\mathcal{B}}}=\left(c_{0}-\partial_{n}\right) \tilde{\boldsymbol{\Phi}}-\lambda_{0}\left[\boldsymbol{U}_{2}+c_{0} \delta(0)\right] \partial_{n} \tilde{\boldsymbol{\phi}} \\
+\lambda_{0} f_{0} \tilde{\boldsymbol{\phi}} \times\left(c_{0}-\partial_{n}\right) \boldsymbol{\phi}
\end{gathered}
$$




$$
\begin{gathered}
\mathcal{J}_{\tilde{\phi_{\mathcal{B}}}}=\lambda_{0} \partial_{n}\left(2 \tilde{\boldsymbol{\phi}}-\mathcal{H}_{\phi}\right)+\lambda_{0} f_{0} \boldsymbol{\phi} \times \partial_{n} \boldsymbol{\phi}, \\
\mathcal{J}_{\partial_{n} \phi}=\lambda_{0} \delta(0) \partial_{n} \tilde{\boldsymbol{\phi}} \\
\mathcal{J}_{\partial_{n} \tilde{\boldsymbol{\phi}}}=\lambda_{0} \delta(0)\left(\partial_{n}-c_{0}\right) \boldsymbol{\phi} \\
\mathcal{J}_{(\triangle \boldsymbol{\phi})_{\mathcal{B}}}=\lambda_{0} \partial_{n} \tilde{\boldsymbol{\phi}}
\end{gathered}
$$

and

$$
\mathcal{J}_{(\triangle \tilde{\phi})_{\mathcal{B}}}=\lambda_{0}\left(\partial_{n}-c_{0}\right) \phi
$$

The subscript $\mathcal{B}$ at local quantities indicates their restriction to the boundary. Further, $\boldsymbol{U}_{2}$ is the matrix of second derivatives of the $|\phi|^{4}$ potential:

$$
U_{2}^{\alpha \beta}=\tau_{0} \delta^{\alpha \beta}+\frac{u_{0}}{6}\left(\delta^{\alpha \beta}+2 \phi^{\alpha} \phi^{\beta}\right),
$$

and for convenience, we have introduced the field

$$
\tilde{\boldsymbol{\Phi}} \equiv \mathcal{R}^{\mathrm{T}} \cdot \tilde{\boldsymbol{\phi}}=\lambda_{0}\left(-\triangle \tilde{\boldsymbol{\phi}}+f_{0} \tilde{\boldsymbol{\phi}} \times \boldsymbol{\phi}\right)
$$

where $\mathcal{R}^{\mathrm{T}}$ denotes the transposed reaction operator, i.e., $\left(\mathcal{R}^{\mathrm{T}}\right)^{\alpha \beta}=\mathcal{R}^{\beta \alpha}$. We shall refer to $\tilde{\boldsymbol{\Phi}}$ as response field since this is its known physical significance包国 (as can be read off again from our Eqs. (41) and (42) below); it should not be confused with the auxiliary field $\tilde{\phi}$.

For simplicity, let us include here in the source part of the action merely bulk sources $\boldsymbol{J}(\boldsymbol{x}, t)$ and $\tilde{\boldsymbol{J}}(\boldsymbol{x}, t)$ as well as surface sources $\boldsymbol{J}_{1}\left(\boldsymbol{x}_{\|}, t\right)$ and $\tilde{\boldsymbol{J}}_{1}\left(\boldsymbol{x}_{\|}, t\right)$ which couple to $\boldsymbol{\phi}, \tilde{\boldsymbol{\phi}}$, $\phi_{\mathcal{B}}$, and $\tilde{\phi}_{\mathcal{B}}$, respectively. Owing to the arbitrariness of $\delta \phi$ and $\delta \tilde{\phi}$, it follows from the above results that the 'equations of motion'

$$
\begin{aligned}
& \mathcal{J}_{\phi}(\boldsymbol{x}, t)=\boldsymbol{J}(\boldsymbol{x}, t), \quad \boldsymbol{x} \notin \mathcal{B}, \\
& \mathcal{J}_{\tilde{\boldsymbol{\phi}}}(\boldsymbol{x}, t)=\tilde{\boldsymbol{J}}(\boldsymbol{x}, t), \quad \boldsymbol{x} \notin \mathcal{B},
\end{aligned}
$$

and the 'boundary equations of motion'

$$
\begin{aligned}
& \mathcal{J}_{\rho}\left(\boldsymbol{x}_{\|} f, t\right)=\boldsymbol{J}_{1}\left(\boldsymbol{x}_{\|}, t\right) \delta_{\rho, \phi_{\mathcal{B}}}+\tilde{\boldsymbol{J}}_{1}\left(\boldsymbol{x}_{\|}, t\right) \delta_{\rho, \tilde{\boldsymbol{\phi}}_{\mathcal{B}}}, \\
& \rho=\boldsymbol{\phi}_{\mathcal{B}}, \tilde{\boldsymbol{\phi}}_{\mathcal{B}}, \partial_{n} \boldsymbol{\phi}, \partial_{n} \tilde{\boldsymbol{\phi}},(\triangle \boldsymbol{\phi})_{\mathcal{B}},(\triangle \tilde{\boldsymbol{\phi}})_{\mathcal{B}},
\end{aligned}
$$


hold inside of averages with the action $\mathcal{J}+\mathcal{J}_{j}$. From the latter and Eqs. (27)-(32), we get the previously given boundary conditions for $\phi$, Eqs. (11) and (16), and two additional ones for $\tilde{\phi}$ : namely

$$
\partial_{n} \tilde{\phi}=0
$$

and

$$
\begin{aligned}
\left(\partial_{n}-c_{0}\right) \tilde{\boldsymbol{\Phi}} & = \\
\left(\partial_{n}-c_{0}\right) \lambda_{0}\left(-\Delta \tilde{\boldsymbol{\phi}}+f_{0} \tilde{\boldsymbol{\phi}} \times \boldsymbol{\phi}\right) & =0 .
\end{aligned}
$$

\section{Fluctuation-dissipation theorem}

The significance of the boundary conditions (38) and (39) can be understood as follows. Let us add a (possibly time-dependent) magnetic field term to the Hamiltonian (6), making the replacement

$$
\mathcal{H} \rightarrow \mathcal{H}_{\boldsymbol{h}}=\mathcal{H}-\int_{\mathbb{R}_{+}^{d}} \boldsymbol{h} \cdot \boldsymbol{\phi},
$$

where we assume $\boldsymbol{h}(\boldsymbol{x}, t)$ to have support only off the surface. This induces the change

$$
\mathcal{J} \rightarrow \mathcal{J}_{\boldsymbol{h}}=\mathcal{J}-\int_{\mathbb{R}_{+}^{d}} \boldsymbol{h} \cdot \tilde{\boldsymbol{\Phi}}
$$

of the dynamic action. Hence we recover the usual correspondence (known from the bulk case 22 )

$$
\frac{\delta}{\delta \boldsymbol{h}(\boldsymbol{x}, t)} \leftrightarrow \tilde{\boldsymbol{\Phi}}(\boldsymbol{x}, t)
$$

between functional derivatives with respect to $\boldsymbol{h}(\boldsymbol{x})$, taken at $\boldsymbol{h}=\mathbf{0}$, and insertions of the response operator on the right-hand side.

Furthermore, the fluctuation-dissipation relation

$$
-\theta\left(t-t^{\prime}\right)\left\langle\dot{\phi}^{\alpha}(\boldsymbol{x}, t) \boldsymbol{\phi}^{\beta}\left(\boldsymbol{x}^{\prime}, t^{\prime}\right)\right\rangle=\left\langle\phi^{\alpha}(\boldsymbol{x}, t) \tilde{\Phi}^{\beta}\left(\boldsymbol{x}^{\prime}, t^{\prime}\right)\right\rangle
$$

can be derived as in Refs. 21, 22, 24, and 32, owing to the form (19) of the action.

The significance of the boundary condition (39) becomes clear if we let $\boldsymbol{x}^{\prime}$ in Eq. (43) approach a point on the boundary plane $\mathcal{B}$ : it ensures the consistency of the fluctuationdissipation theorem (43) with the boundary condition (11). 
To understand the Neumann boundary condition (38), note first that according to Eq. (19), the reaction operator couples $\nabla \tilde{\phi}$ to the current operator $-\lambda_{0} \nabla \delta \mathcal{H} / \delta \phi$. This boundary condition ensures that the reaction operator is self-adjoint. In addition, it can be combined with Eq. (28) to see that the boundary equation of motion (37) for $\rho=\tilde{\phi}_{\mathcal{B}}$ leads back to the boundary conditions (16) for the currents.

\section{RG ANALYSIS OF THE SEMI-FINITE MODEL J}

\section{A. Preliminaries}

We now turn to the RG analysis of the semi-infinite model $\mathrm{J}$ introduced in the previous section. To this end, two RG schemes will be used: a massless one based on minimal subtraction of poles and the expansion about six dimensions, called $\mathrm{RS}_{1}$, and a massive one for fixed dimensions $2<d \leq 4$, called $\mathrm{RS}_{2}$.

Before embarking on a discussion of either one of these, we must set up some notation. Let us define the generating functionals of (connected) correlation and response functions

$$
\begin{aligned}
& \mathcal{W}\left[\tilde{\boldsymbol{J}}, \boldsymbol{J}, \boldsymbol{K} ; \tilde{\boldsymbol{J}}_{1}, \boldsymbol{J}_{1}\right] \\
& =\ln \left\langle e^{(\tilde{\boldsymbol{J}}, \tilde{\boldsymbol{\phi}})+(\boldsymbol{J}, \boldsymbol{\phi})+(\boldsymbol{K}, \tilde{\boldsymbol{\phi}} \times \boldsymbol{\phi})+\left(\tilde{\boldsymbol{J}}_{1}, \tilde{\boldsymbol{\phi}}_{\mathcal{B}}\right)+\left(\boldsymbol{J}_{1}, \boldsymbol{\phi}_{\mathcal{B}}\right)}\right\rangle
\end{aligned}
$$

and

$$
\begin{aligned}
& \mathcal{G}\left[\tilde{\boldsymbol{J}}, \boldsymbol{J} ; \tilde{\boldsymbol{J}}_{1}, \boldsymbol{J}_{1}\right] \\
& =\ln \left\langle e^{(\tilde{\boldsymbol{J}}, \tilde{\boldsymbol{\Phi}})+(\boldsymbol{J}, \boldsymbol{\phi})+\left(\tilde{\boldsymbol{J}}_{1}, \tilde{\boldsymbol{\Phi}}_{\mathcal{B}}\right)+\left(\boldsymbol{J}_{1}, \boldsymbol{\phi}_{\mathcal{B}}\right)}\right\rangle
\end{aligned}
$$

where we have introduced the convenient short-hands

$$
(\boldsymbol{J}, \boldsymbol{\phi}) \equiv \int_{-\infty}^{\infty} d t \int_{\mathbb{R}_{+}^{d}} d^{d} x \boldsymbol{J}(\boldsymbol{x}, t) \cdot \boldsymbol{\phi}(\boldsymbol{x}, t)
$$

and

$$
\left(\boldsymbol{J}_{1}, \boldsymbol{\phi}_{\mathcal{B}}\right) \equiv \int_{-\infty}^{\infty} d t \int_{\mathcal{B}} d^{d-1} x_{\|} \boldsymbol{J}_{1}\left(\boldsymbol{x}_{\|}, t\right) \cdot \boldsymbol{\phi}_{\mathcal{B}}\left(\boldsymbol{x}_{\|}, t\right)
$$

For the cumulants generated by these functionals we write

$$
\begin{aligned}
& W^{(\tilde{N}, N, L ; \tilde{M}, M)} \\
& =\left\langle\prod_{j=1}^{\tilde{N}} \tilde{\phi}^{\tilde{\alpha}_{j}} \prod_{k=1}^{N} \phi^{\alpha_{k}} \prod_{l=1}^{L}(\tilde{\phi} \times \boldsymbol{\phi})^{\gamma_{l}} \prod_{m=1}^{\tilde{M}} \tilde{\phi}_{\mathcal{B}}^{\tilde{\beta}_{m}} \prod_{n=1}^{M} \phi_{\mathcal{B}}^{\beta_{n}}\right\rangle^{\mathrm{cum}}
\end{aligned}
$$


and

$$
\begin{aligned}
& G^{(\tilde{N}, N ; \tilde{M}, M)} \\
& =\left\langle\prod_{j=1}^{\tilde{N}} \tilde{\Phi}^{\tilde{\alpha}_{j}} \prod_{k=1}^{N} \phi^{\alpha_{k}} \prod_{m=1}^{\tilde{M}} \tilde{\Phi}_{\mathcal{B}}^{\tilde{\beta}_{m}} \prod_{n=1}^{M} \phi_{\mathcal{B}}^{\beta_{n}}\right\rangle^{\mathrm{cum}} .
\end{aligned}
$$

We normally suppress the tensorial indices $\tilde{\alpha}_{1}, \ldots, \beta_{M}$ of these functions, but give their space and time (or momentum and frequency) coordinates (suppressed above) when dealing with specific ones.

The reason for considering the functions $W^{(\tilde{N}, N, L ; \tilde{M}, M)}$ should be clear: aside from multipoint cumulants of the basic fields $\tilde{\phi}$ and $\phi$, insertions of the composite operator $\tilde{\phi} \times \phi$ are needed because it appears in the fluctuation-dissipation relation (43).

The free response propagator and free correlator one needs to compute the $W$ and $G$ functions defined above are the same as for model $\mathrm{B}_{\mathrm{B}}$, and for the case $\tau_{0}>0$, may be gleaned from any of the Refs. 8,8.9. In a mixed $\boldsymbol{p} z \omega$ representation (where $\boldsymbol{p} \in \mathbb{R}^{d-1}$ is a $(d-1)$-dimensional wave-vector conjugate to $\boldsymbol{x}_{\|}$while $\omega$ denotes the Fourier frequency variable associated with $t$ ), the free response propagator reads

$$
\begin{aligned}
\hat{G}(\boldsymbol{p} ; z, \tilde{z} ; \omega)=\frac{1}{2 \lambda_{0}} & \left(\frac{\tau_{0}}{4}+i \frac{\omega}{\lambda_{0}}\right)^{-1 / 2}\left\{\frac{1}{2 \kappa_{-}}\left[e^{-\kappa_{-}|z-\tilde{z}|}-f_{-} e^{-\kappa_{-}(z+\tilde{z})}-g_{-} e^{-\left(\kappa_{-} z+\kappa_{+} \tilde{z}\right)}\right]\right. \\
& \left.-\frac{1}{2 \kappa_{+}}\left[e^{-\kappa_{+}|z-\tilde{z}|}-f_{+} e^{-\kappa_{+}(z+\tilde{z})}-g_{+} e^{-\left(\kappa_{+} z+\kappa_{-} \tilde{z}\right)}\right]\right\}
\end{aligned}
$$

with

$$
f_{ \pm} \equiv f_{ \pm}\left(\kappa_{ \pm}, \kappa_{\mp} ; c_{0}, \kappa\right)=\frac{\kappa_{ \pm} \kappa_{\mp}\left(\kappa_{ \pm}^{2}-\kappa_{\mp}^{2}\right)-c_{0}\left[\kappa_{ \pm}\left(\kappa^{2}-\kappa_{ \pm}^{2}\right)+\kappa_{\mp}\left(\kappa^{2}-\kappa_{\mp}^{2}\right)\right]}{\kappa_{ \pm}\left(\kappa^{2}-\kappa_{ \pm}^{2}\right)\left(c_{0}+\kappa_{\mp}\right)-\kappa_{\mp}\left(\kappa^{2}-\kappa_{\mp}^{2}\right)\left(c_{0}+\kappa_{ \pm}\right)}
$$

and

$$
g_{ \pm} \equiv g_{ \pm}\left(\kappa_{ \pm}, \kappa_{\mp} ; c_{0}, \kappa\right)=\frac{2 c_{0} \kappa_{ \pm}\left(\kappa^{2}-\kappa_{\mp}^{2}\right)}{\kappa_{ \pm}\left(\kappa^{2}-\kappa_{ \pm}^{2}\right)\left(c_{0}+\kappa_{\mp}\right)-\kappa_{\mp}\left(\kappa^{2}-\kappa_{\mp}^{2}\right)\left(c_{0}+\kappa_{ \pm}\right)} .
$$

Here

$$
\kappa \equiv+\sqrt{\boldsymbol{p}^{2}+\tau_{0}}
$$

and $\kappa_{ \pm}$denote the roots with positive real parts of the equation

$$
\kappa_{ \pm}^{2}=\boldsymbol{p}^{2}+\left(\tau_{0} / 2\right) \pm\left[\left(\tau_{0} / 2\right)^{2}+i\left(\omega / \lambda_{0}\right)\right]^{1 / 2}
$$


The free correlator $C \delta^{\alpha \beta}=\left\langle\phi^{\alpha} \phi^{\beta}\right\rangle^{\text {cum }}$ can be expressed in terms of the free response propagator as

$$
\begin{aligned}
\hat{C}\left(\boldsymbol{p} ; z, z^{\prime} ; \omega\right)=2 \lambda_{0} \int_{0}^{\infty} & d \tilde{z} \hat{G}(\boldsymbol{p} ; z, \tilde{z} ; \omega) \\
& \times\left(\boldsymbol{p}^{2}-\partial_{\tilde{z}}^{2}\right) \hat{G}\left(-\boldsymbol{p} ; z^{\prime}, \tilde{z} ;-\omega\right) .
\end{aligned}
$$

Owing to the presumed $O(3)$ symmetry of the Hamiltonians (11) and (6) of our lattice and continuum models, the only surface transition that is possible in three dimensions is the ordinary transition. We can benefit from the fact that its asymptotic critical behavior can be studied by taking the limit $c_{0} \rightarrow \infty$ (see, e.g., Ref. 5 and below). The simplified expressions for the free response propagator and correlator which then apply correspond to the replacements of the coefficients $f_{ \pm}$and $g_{ \pm}$by

$$
f_{ \pm}^{\infty} \equiv \lim _{c_{0} \rightarrow \infty} f_{ \pm}\left(\kappa_{ \pm}, \kappa_{\mp} ; c_{0}, \kappa\right)=-\frac{\kappa_{ \pm}-\kappa_{\mp}}{\kappa_{ \pm}+\kappa_{\mp}}
$$

and

$$
g_{ \pm}^{\infty} \equiv \lim _{c_{0} \rightarrow \infty} g_{ \pm}\left(\kappa_{ \pm}, \kappa_{\mp} ; c_{0}, \kappa\right)=-\frac{2 \kappa_{ \pm}}{\kappa_{ \pm}+\kappa_{\mp}}
$$

respectively.

\section{B. Massless renormalization scheme near six dimensions $\left(\mathrm{RS}_{1}\right)$}

We here restrict ourselves to bulk dimensions $4<d \leq 6$. Then the static critical behavior is described by Landau theory. The Gaussian fixed point, $u_{0}=0$, of the $|\phi|^{4}$ theory is infrared-stable. In part of the calculation $u_{0}$ therefore can be set to zero. This is possible as long as we consider quantities that have a nonsingular and nonvanishing limit $u_{0} \rightarrow 0$. However, we must keep in mind that the linear scaling field $u$ associated with $u_{0}$ is dangerous

irrelevant 136 Quantities like the free energy density or the spontaneous magnetization vary as inverse powers of $u_{0} \sim u$ for $u_{0} \rightarrow 0$, and hyperscaling is broken. Accordingly, already a full scaling description of the static bulk critical behavior requires the inclusion of a second, so-called thermodynamic, length besides the bulk correlation length. Finally, in applying techniques of renormalized field theory, we must remember that both the static as well as the dynamic theories are not renormalizable for $d>4$ if $u_{0} \neq 0$. Single insertions of the 
local operator to which $u_{0}$ couples can be renormalized, but the additional counterterms to which it gives rise are not sufficient for curing the additional ultraviolet (uv) singularities produced by multiple insertions.

We now consider the $W$ functions (49), where we restrict the temperature $T$ to values above the critical temperature $T_{\mathrm{c}}$ and set $u_{0}=0$ temporarily. Since the Hamiltonian (6) becomes Gaussian for $u_{0}=0$, there are no static uv singularities to cure. Hence no amplitude renormalization of the order parameter is required, i.e., $Z_{\phi}=1$. By power counting one finds that counterterms of the form $\int d t \int_{\mathbb{R}_{+}^{d}} \tilde{\phi} \cdot \dot{\phi}$ are also not needed. Since this implies that the product of amplitude renormalization factors $Z_{\phi} Z_{\tilde{\phi}}$ for $\phi$ and $\tilde{\phi}$ is unity, an amplitude renormalization of $\tilde{\phi}$ is not required either $\left(Z_{\tilde{\phi}}=1\right.$ ). A (bulk) counterterm $\propto \phi \cdot \triangle \tilde{\phi}$ is ruled out for $u_{0}=0$. If $u_{0}$ did not vanish, the $O\left(u_{0}\right)$ contribution to such a counterterm would diverge as $\Lambda^{4}$ for $d=6$ within a theory regularized by a cutoff $\Lambda$.

We assert that the following counterterms are sufficient to renormalize the generating functional $\mathcal{W}$ : aside from those implied by the reparametrizations

$$
\lambda_{0}=\mu^{-4} Z_{\lambda}\left(f^{2}, d\right) \lambda
$$

and

$$
f_{0}=\mu^{\frac{6-d}{2}}\left[Z_{\lambda}\left(f^{2}, d\right)\right]^{-1} f
$$

only a counterterm of the form $\int d t \int_{\mathbb{R}_{+}^{d}} \boldsymbol{K} \cdot \triangle \tilde{\boldsymbol{\phi}}$ is required, where $\mu$ is an arbitrary momentum scale. More precisely, we claim that the cumulants generated by the functional

$$
\mathcal{W}_{\text {ren }}\left[\tilde{\boldsymbol{J}}, \boldsymbol{J}, \boldsymbol{K} ; \tilde{\boldsymbol{J}}_{1}, \boldsymbol{J}_{1}\right]=\mathcal{W}\left[\tilde{\boldsymbol{J}}, \boldsymbol{J}, \boldsymbol{K} ; \tilde{\boldsymbol{J}}_{1}, \boldsymbol{J}_{1}\right]-\left(Z_{\lambda}-1\right) f^{-1} \mu^{-\frac{6-d}{2}} \int_{-\infty}^{\infty} d t \int_{\mathbb{R}_{+}^{d}} \boldsymbol{K} \cdot \triangle \tilde{\boldsymbol{\phi}}
$$

are $u v$ finite when expressed in terms of $\lambda, f$ (and $c_{0}$ or its dimensionless equivalent $c \equiv c_{0} / \mu$ ).

This conclusion is based on the following observations. The detailed-balance form of the action (19) in conjunction with the constraints imposed by the conservation of the order parameter and power counting restricts the possible bulk counterterms to those included in Eq. (60). Using this result as input, one can consider the renormalization of the bulk analog of the fluctuation-dissipation relation (43). For convenience we employ dimensional regularization and fix the counterterms by minimal subtraction of poles at $d=6$. From the uv finiteness of the correlation function of the renormalized function on the left-hand 
side of this equation two conclusions may then be drawn: the renormalization factors of $f_{0}$ and $\lambda_{0}$ are reciprocal to each other, and the renormalization function of the $\boldsymbol{K}$-dependent counterterm is related to $Z_{\lambda}$ in the stated fashion. 37 The result means that insertions of the response field (34) renormalize just as $\phi$, requiring no additional counterterms.

A final step remains to complete the argument: we must show that no further surface counterterms are needed. Given the causal structure of the theory (according to which at least one $\tilde{\phi}$ must occur in any monomial of the action), power counting restricts the possible candidates for such counterterms in $d=6$ dimensions to boundary contributions to the action involving monomials of the form $\lambda_{0} \tilde{\phi}^{2}, \lambda_{0} \tilde{\phi} \cdot \boldsymbol{\phi}$, and $\lambda_{0} \tilde{\phi}^{\alpha} \phi^{\beta} \phi^{\gamma}$ (as well as similar ones with derivatives), where the coefficients have momentum dimensions 1,3 , and 0 , respectively. Now the cubic nonlinearity of the bare action can be rewritten as

$$
\mathcal{J}_{\mathrm{mcv}}=\lambda_{0} f_{0} \epsilon^{\alpha \beta \gamma} \int_{-\infty}^{\infty} d t \int_{\mathbb{R}_{+}^{d}}\left(\nabla \tilde{\phi}^{\alpha}\right) \phi^{\beta} \nabla \phi^{\gamma}
$$

upon integrating by parts and utilizing the boundary condition (11). Thus each $\tilde{\phi}$ leg of the vertex $\propto f_{0}$ comes with a derivative $\boldsymbol{\nabla}$. This reduces the superficial degree of divergence of such uv boundary singularities with two $\tilde{\phi}$ legs by two, making it negative (uv superficially convergent). By a similar argument, surface counterterms involving one $\tilde{\phi}$ and two $\phi$ fields are ruled out. Hence we are left with surface counterterms $\propto \phi \cdot \nabla \tilde{\phi}$ and analogous ones with up to two additional derivatives.

To proceed we follow Ref. 22 and perform the integral $\int_{-\infty}^{\infty} d t$ of the fluctuation-dissipation relation (43). This yields

$$
\left\langle\phi^{\alpha}(\boldsymbol{x}) \phi^{\beta}\left(\boldsymbol{x}^{\prime}\right)\right\rangle^{\mathrm{st}}=-\lambda_{0} \Delta^{\prime}\left\langle\phi^{\alpha}(\boldsymbol{x}) \tilde{\phi}^{\beta}\left(\boldsymbol{x}^{\prime}\right)\right\rangle_{\omega=0}+\lambda_{0} f_{0}\left\langle\phi^{\alpha}(\boldsymbol{x})(\tilde{\boldsymbol{\phi}} \times \boldsymbol{\phi})^{\beta}\left(\boldsymbol{x}^{\prime}\right)\right\rangle_{\omega=0}
$$

where the superscript 'st' indicates a static quantity while the subscript $\omega$ at the expectation values on the right-hand side means their Fourier transform with respect to the time difference $t-t^{\prime}$. We multiply this equation from the right by the inverse of the static propagator on the right-hand side and from the left with the vertex function $\Gamma_{\tilde{\phi}^{\alpha} \phi^{\beta}}$. The result is 38

$$
\Gamma_{\tilde{\phi}^{\alpha} \phi^{\beta}}\left(\boldsymbol{x}, \boldsymbol{x}^{\prime} ; \omega=0\right)=\lambda_{0}\left(-\triangle^{\prime}+\tau_{0}\right)\left[-\triangle \delta\left(\boldsymbol{x}-\boldsymbol{x}^{\prime}\right)+f_{0} \Gamma_{\tilde{\phi}^{\alpha} ;(\tilde{\boldsymbol{\phi}} \times \boldsymbol{\phi})^{\beta}}\left(\boldsymbol{x}, \boldsymbol{x}^{\prime} ; \omega=0\right)\right]
$$

where $\Gamma_{\tilde{\phi}^{\alpha} ;(\tilde{\phi} \times \phi)^{\beta}}$ means a vertex functions with a single $\tilde{\phi}^{\alpha}$ leg and an insertion of the composite operator $(\tilde{\phi} \times \phi)^{\beta}$. Owing to the operator $-\triangle^{\prime}+\tau_{0}$ (inverse static propagator) on the 
right-hand side, the uv behavior of possible primitive local divergences of the vertex function on the left-hand side is improved by two powers of $\Lambda$. This is sufficient to ensure that

no additional surface counterterms with one $\tilde{\phi}$ and one $\phi$ leg are needed. Since the vertex function $\Gamma_{\tilde{\phi}^{\alpha} ;(\tilde{\boldsymbol{\phi}} \times \boldsymbol{\phi})^{\beta}}$ has an explicit $\boldsymbol{\nabla}$ acting on the external leg, it has a primitive logarithmic bulk singularity that is cured by the subtraction provided by the bulk counterterm $\propto \boldsymbol{K} \cdot \Delta \tilde{\boldsymbol{\phi}}$. On dimensional grounds one might anticipate logarithmically divergent surface counterterms of the form $\left(\partial_{n} \tilde{\boldsymbol{\phi}}\right) \cdot\left(\tau_{0}-\triangle\right) \boldsymbol{\phi}$ and $\tilde{\boldsymbol{\phi}} \cdot \partial_{n}\left(\tau_{0}-\triangle\right) \boldsymbol{\phi}$, but these are annihilated by the boundary conditions (38) and (16), respectively. Note also that the restriction to $\omega=0$ is unproblematic here because each factor of $\omega$ (i.e., each time derivative) reduces the superficial degree of divergence by four.

A further comment is appropriate here. Field theories for systems with boundaries are known to have the following feature: Besides one-particle irreducible (1PI) graphs, oneparticle reducible (1PR) ones may also require 'final subtractions' and hence contribute to renormalization functions (cf. Sec. II.B.6 of Ref. 5). Nevertheless our above reasoning based on 1PI graphs is conclusive since the power counting would not be changed if we contracted 1PR graphs whose external free legs are amputated to a point. Hence the counterterms included in $\mathcal{W}_{\text {ren }}$ are indeed sufficient to cure the uv singularities of the $W$ functions (48). The uv finiteness of the $G$ functions generated by

$$
\mathcal{G}_{\text {ren }}\left[\tilde{\boldsymbol{J}}, \boldsymbol{J}, \boldsymbol{K} ; \tilde{\boldsymbol{J}}_{1}, \boldsymbol{J}_{1}\right]=\mathcal{G}\left[\tilde{\boldsymbol{J}}, \boldsymbol{J}, \boldsymbol{K} ; \tilde{\boldsymbol{J}}_{1}, \boldsymbol{J}_{1}\right]
$$

when expressed in terms of $\lambda$ and $f$ (as well as $\tau_{0}$ and $c_{0}$ or their dimensionless equivalents $\tau \equiv \tau_{0} / \mu^{2}$ and $\left.c \equiv c_{0} / \mu\right)$ follows as a simple corollary from the fact that the response field $\tilde{\boldsymbol{\Phi}}$ renormalizes just as $\phi$ (namely trivially).

\section{RG analysis in $6-\epsilon$ dimensions}

Utilizing the results of the previous subsection, one can perform a RG analysis of quantities that are finite and nonzero for $u_{0}=0$. This criterion is satisfied by both the $W$ and the $G$ functions for $\tau_{0}>0$, as can be checked via perturbation theory in $f_{0}$. Since such a RG analysis is standard we can be brief and just state its principal results.

To one-loop order the renormalization function $Z_{\lambda}$ is given by 39

$$
Z_{\lambda}(f)=1-\frac{1}{192 \pi^{3}} \frac{f^{2}}{6-d}+O\left(f^{4}\right) .
$$


Equation (59) implies that the beta function

$$
\left.\beta_{f}(f) \equiv \mu \partial_{\mu}\right|_{0} f
$$

can be written in terms of the exponent function

$$
\left.\eta_{\lambda}(f) \equiv \mu \partial_{\mu}\right|_{0} \ln Z_{\lambda}
$$

namely

$$
\beta_{f}(f)=-f\left[\frac{6-d}{2}-\eta_{\lambda}(f)\right],
$$

where $\left.\partial_{\mu}\right|_{0}$ denotes the derivative at fixed bare coupling constants $f_{0}, \tau_{0}$, and $c_{0}$. The infrared-stable fixed point $f^{*}$ for $4 \leq d<6$ is given by the nontrivial root of the equation $\beta_{f}\left(f^{*}\right)=0$. From Eq. (68) we find for the value of the exponent function $\eta_{\lambda}^{*}$ at the fixed point $f^{*}$ the result

$$
\eta_{\lambda}^{*} \equiv \eta_{\lambda}\left(f^{*}\right)=\frac{6-d}{2}
$$

which we insert into the general expression

$$
\mathfrak{z}=4-\eta_{\lambda}^{*}
$$

for the dynamic critical exponent $\mathfrak{z}$. Since the correlation exponent $\eta$ is zero for $d \geq 4$, the final result $\mathfrak{z}=(d+2) / 2$ for $4 \leq d \leq 6$ is consistent with the established value $19,20,21,22,23,40$

$$
\mathfrak{z}=\frac{d+2-\eta}{2}, \quad 2<d \leq 6 .
$$

The latter result is known to follow most easily from the observation that the characteristic frequency of isotropic ferromagnets for $T>T_{\mathrm{c}}$ is the Larmor frequency and hence scales as the (static) bulk magnetic field $\boldsymbol{h} .40,4$

Next we turn to the analysis of the critical behavior of surface quantities. We restrict the following discussion to the case of the ordinary transition, the only surface transition at bulk criticality that remains in the physically interesting three-dimensional case. 2243 The surface enhancement variable $c \equiv c_{0} / \mu$ transforms as $c \rightarrow \bar{c}(l)=c / \ell$ under scale transformations $\mu \rightarrow \mu \ell$ and hence approaches the fixed-point value $c_{\text {ord }}^{*}=\infty$ in the infrared limit $\ell \rightarrow 0$, provided its initial value is positive. In this case we can set $c_{0}=\infty$ from the outset. Surface quantities involving the surface fields $\phi_{\mathcal{B}}$ or $\tilde{\boldsymbol{\Phi}}_{\mathcal{B}}$ then vanish. 
Let $F_{\text {ren }}$ be any of the two renormalized functions $W_{\text {ren }}^{(\tilde{N}, N, L ; \tilde{M}, M)}$ and $G_{\text {ren }}^{(\tilde{N}, N ; \tilde{M}, M)}$ generated by the functionals (60) and (64) for $u_{0}=0$ and $\tau \equiv \tau_{0} / \mu^{2} \geq 0$. The invariance of the regularized bare functions with respect to a change of $\mu$ implies the $\mathrm{RG}$ equation

$$
\left[\mu \partial_{\mu}+\beta_{f} \partial_{f}-2 \tau \partial_{\tau}+\left(4-\eta_{\lambda}\right) \lambda \partial_{\lambda}-c \partial_{c}\right] F_{\text {ren }}=0 .
$$

This may be utilized in a familiar manner to obtain the asymptotic scaling forms of these functions. The result one obtains for the pair correlation function $C^{\alpha \beta}=\delta^{\alpha \beta} C\left(=G^{(0,2 ; 0,0)}\right)$ at $T=T_{\mathrm{c}}$ agrees with the more general one predicted in our previous paper,18

$$
C\left(\boldsymbol{r} ; z, z^{\prime} ; t\right) \approx r^{2-d-\eta} \Upsilon\left(z / r, z^{\prime} / r ; t r^{-\mathfrak{z}}\right)
$$

if the classical value $\eta=0$ and the implied one $\mathfrak{z}=(d+2) / 2$ are substituted (as is appropriate for $4<d<6$ ). Here we have suppressed the variables $\mu$ and $\lambda$, setting both to unity. The variable $c$ does not appear on the right-hand side because the scaling function $\Upsilon$ is a property of the $c=\infty$ fixed point. Deviations of $c$ from the value $c=\infty$ produce corrections to the displayed leading infrared contribution.

The derivation of the scaling form of the surface correlation function

$$
C_{11}(\boldsymbol{r} ; t) \equiv C(\boldsymbol{r} ; 0,0 ; t)
$$

is not quite so straightforward because $\left.C\right|_{c=\infty}$ vanishes whenever $z=0$ or $z^{\prime}=0$, as a consequence of the Dirichlet boundary condition into which Eq. (11) turns for $c_{0}=\infty$. One possibility to cope with this difficulty is to the study behavior of $C_{11}$ in the limit $c \rightarrow \infty$. As is expounded elsewhere, 明明幽 this can be achieved by an expansion in powers of $1 / c_{0}$. According to the boundary conditions (11) and (39), the boundary operators $\phi_{\mathcal{B}}$ and $\tilde{\boldsymbol{\Phi}}_{\mathcal{B}}$ can be replaced by $c_{0}^{-1} \partial_{n} \boldsymbol{\phi}_{\mathcal{B}}$ and $c_{0}^{-1} \partial_{n} \tilde{\boldsymbol{\Phi}}_{\mathcal{B}}$, respectively. From previous detailed investigations correlation and response functions involving these boundary operators take at the ordinary transition can be determined: making the replacements $\boldsymbol{\phi}_{\mathcal{B}} \rightarrow \partial_{n} \boldsymbol{\phi}_{\mathcal{B}}$ and $\tilde{\boldsymbol{\Phi}}_{\mathcal{B}} \rightarrow \partial_{n} \tilde{\boldsymbol{\Phi}}_{\mathcal{B}}$, one studies the so-obtained analogs of these functions, with $c_{0}$ set to $\infty$.

An alternative strategy, which leads to equivalent results, is to use the boundary operator expansion (BOE). According to Eqs. (11) and (39), both the order parameter density $\phi$ as well as the response field $\tilde{\boldsymbol{\Phi}}$ satisfy Dirichlet boundary conditions if $c_{0}=\infty$. In analogy 
with the static case, 0 国 a BOE of the form

$$
\phi(\boldsymbol{x}, t) \underset{z \rightarrow 0}{\approx} D(z, t) \partial_{n} \boldsymbol{\phi}\left(\boldsymbol{x}_{\mathcal{B}}, t\right)+\ldots
$$

and its counterpart involving $\tilde{\boldsymbol{\Phi}}$ and $\partial_{n} \tilde{\boldsymbol{\Phi}}$ should hold as $\boldsymbol{x}=\left(\boldsymbol{x}_{\|}, z\right)$ approaches the surface point $\boldsymbol{x}_{\mathcal{B}}=\left(\boldsymbol{x}_{\|}, 0\right)$. (We have suppressed contributions proportional to the operator $\mathbb{1}$, which occur when the average $\langle\phi\rangle$ does not vanish.5.46.47) Equation 75 implies that cumulants involving the surface operators $\partial_{n} \phi$ and $\partial_{n} \tilde{\boldsymbol{\Phi}}$ give access to surface correlation functions. We refrain from doing this within the framework of the $\epsilon_{6}$ expansion and turn directly to $\mathrm{RS}_{2}$, the massive RG scheme.

\section{Massive renormalization scheme for $2<d \leq 4\left(\mathrm{RS}_{2}\right)$}

Our aim here is to extend the massive RG scheme for semi-infinite systems developed by one of us and Shpot 25,26 to the dynamic theory of the model-J action (21). We assume that $2<d \leq 4$ and give up the restriction $u_{0}=0$, i.e., both $u_{0}$ and $f_{0}$ are assumed to be nonzero.

The scheme can be extended for general values of $c_{0}$. However, as we are primarily interested in the dynamic surface critical behavior at the ordinary transition in $d=3$ bulk dimensions, we can simplify the analysis by setting $c_{0}=\infty$ from the outset. The advantage of doing this is considerable: for general values of $c_{0}$, the renormalization factors associated with surface operators (called 'surface renormalization factors' for short) depend on the renormalized coupling constant $u$ (to be defined below) and the ratio $c / m$, where $c$ and $m$ are the renormalized analog 18 of the bare surface enhancement $c_{0}$ and the renormalized mass $m$ (to be introduced below), respectively. This additional dependence on the mass ratio $c / m$ makes the RG analysis rather cumbersome. If we set $c_{0}=\infty$, we focus directly on the asymptotic regime $c / m=\infty$ and avoid this difficulty because the surface renormalization factors (which are of purely static origin) can then be chosen to depend merely on $u$.

\section{Static bulk renormalization functions}

Let $\Gamma_{\mathrm{st}, \mathrm{b}}^{(N, I)}$ be the static bulk vertex function with $N$ legs of type $\phi$ and $I$ insertions of $\phi^{2} / 2$, and $\check{\Gamma}_{\mathrm{st}, \mathrm{b}}^{(N, I)}(\boldsymbol{q}, \boldsymbol{Q})$ the Fourier transform of this (translationally invariant) function, up to the momentum-conserving factor $(2 \pi)^{d} \delta\left(\sum \boldsymbol{q}+\sum \boldsymbol{Q}\right)$. Here $\boldsymbol{q}$ and $\boldsymbol{Q}$ are the $N$ and $I$ momenta 
conjugate to the positions $\boldsymbol{x}$ and $\boldsymbol{X}$ of the legs and the inserted operators, respectively. We write

$$
\begin{gathered}
\tau_{0}=m^{2}+\delta m^{2}, \\
\phi=\left[Z_{\phi}(u)\right]^{1 / 2} \phi_{\text {ren }}, \\
\phi^{2}=\left[Z_{\phi^{2}}(u)\right]^{-1}\left[\phi^{2}\right]_{\text {ren }},
\end{gathered}
$$

and

$$
u_{0}=Z_{u}(u) m^{4-d} u
$$

which introduce the renormalized mass $m$, the renormalized densities $\boldsymbol{\phi}_{\mathrm{ren}}$ and $\left[\boldsymbol{\phi}^{2}\right]_{\mathrm{ren}}$, and the renormalized coupling constant $u$.

The mass shift $\delta m^{2}$ and the renormalization (' $Z$ ') factors are fixed via the familiar normalization conditions

$$
\begin{gathered}
\left.\check{\Gamma}_{\text {st }, \mathrm{b}, \mathrm{ren}}^{(2)}(q ; u, m)\right|_{q=0}=m^{2}, \\
\left.\frac{\partial}{\partial q^{2}} \check{\Gamma}_{\mathrm{st}, \mathrm{b}, \mathrm{ren}}^{(2)}(q ; u, m)\right|_{q=0}=1, \\
\left.\check{\Gamma}_{\text {st,b,ren }}^{(2,1)}(\boldsymbol{q}, \boldsymbol{Q} ; u, m)\right|_{\boldsymbol{q}=\boldsymbol{Q}=\mathbf{0}}=1,
\end{gathered}
$$

and

$$
\left.\check{\Gamma}_{\mathrm{st}, \mathrm{b}, \mathrm{ren}}^{(4)}\left(\left\{\boldsymbol{q}_{i}\right\} ; u, m\right)\right|_{\left\{\boldsymbol{q}_{i}=\mathbf{0}\right\}}=m^{4-d} u
$$

for the renormalized static bulk (b) vertex functions

$$
\Gamma_{\mathrm{st}, \mathrm{b}, \mathrm{ren}}^{(N, I)}(\cdot ; m, u)=\left[Z_{\phi}(u)\right]^{N / 2}\left[Z_{\phi^{2}}(u)\right]^{I} \Gamma_{\mathrm{st}, \mathrm{b}}^{(N, I)}\left(\cdot ; \tau_{0}, u_{0}\right)
$$

with $(N, I) \neq(0,1),(0,2)$. 


\section{Dynamic bulk renormalization functions}

We introduce renormalized auxiliary and response fields $\tilde{\phi}_{\text {ren }}$ and $\tilde{\boldsymbol{\Phi}}_{\text {ren }}$, a dynamic bulk renormalization factor $Z_{\lambda}$, and the renormalized bulk variables $\lambda$ and $f$ such that

$$
\begin{gathered}
\tilde{\boldsymbol{\phi}}=\left[Z_{\phi}(u)\right]^{-1 / 2} \tilde{\boldsymbol{\phi}}_{\mathrm{ren}}, \\
\tilde{\boldsymbol{\Phi}}=\left[Z_{\phi}(u)\right]^{1 / 2} \tilde{\boldsymbol{\Phi}}_{\mathrm{ren}}, \\
\lambda_{0}=m^{-4} Z_{\lambda}(u, f) \lambda,
\end{gathered}
$$

and

$$
f_{0}=m^{\frac{6-d}{2}}\left[Z_{\lambda}(u, f)\right]^{-1}\left[Z_{\phi}(u)\right]^{1 / 2} f
$$

To fix the function $Z_{\lambda}$, we choose the normalization condition

$$
\left.\frac{\partial}{\partial q^{2}} \check{\Gamma}_{\tilde{\phi}^{\alpha} \tilde{\phi}^{\beta}}^{(\mathrm{b}, \mathrm{ren})}(q, \omega ; u, f, \lambda, m)\right|_{q=\omega=0}=-2 m^{-4} \lambda \delta^{\alpha \beta},
$$

where $\check{\Gamma}_{\tilde{\phi}^{\alpha} \tilde{\phi}^{\beta}}^{(\text {,ren }}$ denotes a renormalized dynamic bulk vertex function in the momentumfrequency $(\boldsymbol{q} \omega)$ representation.

Let us add a few clarifying remarks. Note, first, that the renormalization functions introduced above are sufficient to absorb the uv singularities of the vertex functions with arbitrary numbers $N$ and $\tilde{N}$ of $\phi$ and $\tilde{\phi}$ legs of the dynamic bulk theory for $d \leq 4$. Hence the above reparametrizations also yield uv finite renormalized functions when applied to the bulk analogs of the $\tilde{N}+N$ point cumulants with $\tilde{N} \tilde{\phi}$ and $N \phi$ fields, i.e., to the bulk analogs of the functions $W^{(\tilde{N}, N, 0 ; 0,0)}$ defined in Eq. (48). The same remark applies to the bulk analogs of the $G$ functions (49).

The meaning of the multiplicative renormalizability of the response field $\tilde{\boldsymbol{\Phi}}$ with respect to the renormalization of the $N+\tilde{N}$ point bulk vertex functions with an insertion of the

composite operator $\tilde{\phi} \times \phi$ has been discussed elsewhere22. 24 and need not be reiterated here: it implies, in particular, that the renormalization of $\Gamma_{\tilde{\phi}^{\alpha} ;(\tilde{\phi} \times \phi)^{\beta}}^{(\mathrm{b})}$ involves a subtraction proportional to $\Gamma_{\tilde{\phi}^{\alpha} ; \triangle \phi^{\beta}}^{(\mathrm{b})}$, chosen in conformity with the multiplicative renormalization (86).

Our second remark concerns the renormalization of $\tilde{\phi}_{\text {ren }}$ and $f$ in Eqs. (85) and (88). The fact that no primitive uv singularities involving a counterterm proportional to $\tilde{\phi}^{\alpha} \dot{\phi}^{\beta}$ 
occur implies that the renormalization factor of $\tilde{\phi}_{\text {ren }}$ is given by $Z_{\phi}^{-1 / 2}$ (up to a uv finite factor). This result means that the latter product of operators transforms according to its engineering dimension under RG transformations and is related to the conservation of the order parameter. 49

The form of the renormalization factor of $f, Z_{\lambda}^{-1} Z_{\phi}^{1 / 2}$, follows from the fluctuationdissipation theorem (43); it tells us that $\dot{\phi}^{\alpha} / \tilde{\Phi}^{\alpha}$ is a RG invariant. As a direct consequence, a relation generalizing Eq. (68) holds between the beta function $\beta_{f}$ and exponent functions, which now are defined via

$$
\left.\beta_{f}(u, f) \equiv m \partial_{m}\right|_{0} f,\left.\quad \beta_{u}(u) \equiv m \partial_{m}\right|_{0} u
$$

and

$$
\left.\eta_{\kappa}(u, f) \equiv m \partial_{m}\right|_{0} \ln Z_{\kappa}, \quad \kappa=\lambda, \phi, \phi^{2}
$$

respectively. We have

$$
\beta_{f}(u, f)=-f\left[\frac{6-d}{2}-\eta_{\lambda}(u, f)+\frac{\eta_{\phi}(u)}{2}\right] .
$$

Since $\eta=\eta_{\phi}\left(u^{*}\right)$, where $u^{*}$ is the nontrivial zero of $\beta_{u}$, this form ensures that the established result (71) for the dynamic exponent $\mathfrak{z}$ is obtained if the value

$$
\eta_{\lambda}^{*} \equiv \eta_{\lambda}\left(u^{*}, f^{*}\right)=\frac{6-d+\eta}{2}
$$

pertaining to the infrared-stable fixed point $\left(u^{*}, f^{*}\right)$ is substituted into Eq. (70).

Finally, let us note that the renormalization factors $Z_{\phi}, Z_{\phi^{2}}, Z_{u}, Z_{\lambda}$ introduced above are all $u v$ finite for $d<4$, although they are logarithmically divergent in the uv if $d=4$ (i.e., they have pole terms at $d=4)$. In other words, if $d<4$, then the uv singularities of the (static and dynamic) bulk theory are absorbed by the mass shift $\delta m^{2}$.

\section{Static surface renormalization functions}

In order to generalize the above approach $\left(\mathrm{RS}_{2}\right)$ to the semi-infinite case, we set $c_{0}=\infty$ and consider the analogs of the $G$ functions (49) that result from these when the boundary operators $\tilde{\boldsymbol{\Phi}}_{\mathcal{B}}$ and $\boldsymbol{\phi}_{\mathcal{B}}$ are replaced by the normal derivatives $\partial_{n} \tilde{\boldsymbol{\Phi}}$ and $\partial_{n} \boldsymbol{\phi}$, respectively. We 
denote these functions as

$$
\begin{aligned}
& G_{\infty}^{(\tilde{N}, N ; \tilde{M}, M)} \\
& \equiv\left\langle\prod_{j=1}^{\tilde{N}} \tilde{\Phi}^{\tilde{\alpha}_{j}} \prod_{k=1}^{N} \phi^{\alpha_{k}} \prod_{m=1}^{\tilde{M}} \partial_{n} \tilde{\Phi}^{\tilde{\beta}_{m}} \prod_{n=1}^{M} \partial_{n} \phi^{\beta_{n}}\right\rangle_{c_{0}=\infty}^{\mathrm{cum}} .
\end{aligned}
$$

Following Refs. 25 and 26, we introduce the static surface renormalization factor $Z_{1, \infty}(u)$ and the renormalized surface operator $\left(\partial_{n} \phi\right)_{\text {ren }}$ via

$$
\partial_{n} \phi=\left[Z_{\phi}(u) Z_{1, \infty}(u)\right]^{1 / 2}\left(\partial_{n} \phi\right)_{\mathrm{ren}} .
$$

Next we take the normal derivative on both sides of the fluctuation-dissipation relation (43) with respect to $\boldsymbol{x}^{\prime}$ and set $t^{\prime}=0$. The result reads

$$
-\theta(t)\left\langle\dot{\phi}^{\alpha}(\boldsymbol{x}, t) \partial_{n} \boldsymbol{\phi}^{\beta}\left(\boldsymbol{x}_{\mathcal{B}}, 0\right)\right\rangle=\left\langle\phi^{\alpha}(\boldsymbol{x}, t) \partial_{n} \tilde{\Phi}^{\beta}\left(\boldsymbol{x}_{\mathcal{B}}, 0\right)\right\rangle
$$

which suggests to renormalize $\partial_{n} \tilde{\boldsymbol{\Phi}}$ in complete analogy with Eq. (95) by

$$
\partial_{n} \tilde{\boldsymbol{\Phi}}=\left[Z_{\phi}(u) Z_{1, \infty}(u)\right]^{1 / 2}\left(\partial_{n} \tilde{\boldsymbol{\Phi}}\right)_{\mathrm{ren}} .
$$

This definition ensures that the modified fluctuation-dissipation relation (96) carries over to the renormalized theory. Moreover, it establishes consistency with the renormalization of the corresponding static correlation function in Ref. 26, provided we fix $Z_{1, \infty}(u)$ as in Eqs. $(7.10 \mathrm{a}-10 \mathrm{~b})$ of that reference. To this end we define the renormalized $G_{\infty}$ functions via

$$
G_{\infty, \text { ren }}^{(\tilde{N}, N ; \tilde{M}, M)}=Z_{\phi}^{-\frac{\tilde{N}+N+\tilde{M}+M}{2}} Z_{1, \infty}^{-\frac{\tilde{M}+M}{2}} G_{\infty}^{(\tilde{N}, N ; \tilde{M}, M)},
$$

if $(\tilde{N}, N ; \tilde{M}, M) \neq(0,0 ; 1,1)$. The excluded function

$$
\begin{aligned}
& \hat{G}_{\infty, \mathrm{ren}}^{(0,0 ; 1,1)}(\boldsymbol{p}, \omega) \\
& =\left[Z_{\phi} Z_{1, \infty}\right]^{-1}\left[\hat{G}_{\infty}^{(0,0 ; 1,1)}(\boldsymbol{p}, \omega)-\hat{G}_{\infty}^{(0,0 ; 1,1)}(\mathbf{0}, 0)\right],
\end{aligned}
$$

requires an additive counterterm, which we choose such that the normalization condition

$$
\left.\hat{G}_{\infty, \text { ren }}^{(0,0 ; 1,1)}(\boldsymbol{p}, \omega)\right|_{p=\omega=0}=0
$$

holds. To specify $Z_{1, \infty}$, we require that

$$
\left.\frac{\partial}{\partial p^{2}} \hat{G}_{\text {ren }}^{(0,0 ; 1,1)}(\boldsymbol{p}, \omega)\right|_{p=\omega=0}=-\frac{1}{2 m} .
$$

Equations (100) and (101) are equivalent to the normalization conditions (7.10a,b) of Ref. 26; together with Eq. (99) they imply that $G^{(0,0 ; 1,1)}$ requires a subtraction and the renormalization factor $Z_{1, \infty}$ is the same as in the static case. 


\section{E. Callan-Symanzik equations}

The Callan-Symanzik (CS) equations can now be derived in a standard fashion. We take a derivative $\partial_{\tau_{0}}$ of the bare (dimensionally regularized) $G_{\infty}$ functions (94) at fixed values of the other bare interaction constants $u_{0}$ and $f_{0}$. Using the above reparametrizations and definitions of the beta and exponent functions then yields

$$
\left[\mathcal{D}_{m}+\frac{\tilde{N}+N+\tilde{M}+M}{2} \eta_{\phi}+\frac{\tilde{M}+M}{2} \eta_{1, \infty}\right] G_{\infty, \mathrm{ren}}^{(\tilde{N}, N ; \tilde{M}, M)}=R_{\infty, \mathrm{ren}}^{(\tilde{N}, N ; \tilde{M}, M)}
$$

with

$$
\mathcal{D}_{m}=m \frac{\partial}{\partial m}+\beta_{u} \frac{\partial}{\partial u}+\beta_{f} \frac{\partial}{\partial f}-\left(4-\eta_{\lambda}\right) \lambda \partial_{\lambda}
$$

and

$$
\begin{aligned}
R_{\infty, \text { ren }}^{(\tilde{N}, N ; \tilde{M}, M)} & \equiv Z_{\phi}^{-(\tilde{N}+N+\tilde{M}+M) / 2} Z_{1, \infty}^{-(\tilde{M}+M) / 2}\left(\left.m \partial_{m}\right|_{0} \tau_{0}\right) \partial_{\tau_{0}} G_{\infty}^{(\tilde{N}, N ; \tilde{M}, M)} \\
& =\left(2-\eta_{\phi}\right) m^{2}\left[\partial_{\tau_{0}} G_{\infty}^{(\tilde{N}, N ; \tilde{M}, M)}\right]_{\mathrm{ren}} .
\end{aligned}
$$

Here the exponent function $\eta_{1, \infty}(u)$ is defined by setting $\kappa=(1, \infty)$ in Eq. (91). Just like the other static functions $\eta_{u}(u), \eta_{\phi}(u), \eta_{\phi^{2}}(u)$, it depends only on $u$ (and $d$ ), but not on the dynamic coupling constant $f$, and is precisely the same as in Ref. 26. The inhomogeneities $R_{\infty, \text { ren }}^{(\tilde{N}, N, \tilde{M}, M)}$ involve renormalized functions with an insertion of $-\int d^{d} x \phi^{2} / 2$, given by

$$
\left[\partial_{\tau_{0}} G_{\infty}^{(\tilde{N}, N ; \tilde{M}, M)}\right]_{\text {ren }}=Z_{\phi}^{-(\tilde{N}+N+\tilde{M}+M) / 2} Z_{1, \infty}^{-(\tilde{M}+M) / 2} Z_{\phi^{2}} \partial_{\tau_{0}} G_{\infty}^{(\tilde{N}, N ; \tilde{M}, M)} .
$$

We proceed along lines similar to those followed, for example, in Refs. 50 and 51, in order to derive the asymptotic scaling forms of the response and correlation functions from the CS equations (102). The deviation $\delta \tau_{0}$ of the bare variable $\tau_{0}$ from its bulk critical value $\tau_{0 \mathrm{c}}$ depends on the temperature difference $\tau \equiv\left(T-T_{\mathrm{c}}\right) / T_{\mathrm{c}}$ according to

$$
\delta \tau_{0} \equiv \tau_{0}-\tau_{0 \mathrm{c}} \sim \tau
$$

which holds if $\tau$ is sufficiently small. Near criticality the mass $m$-i.e., the inverse $\xi^{-1}$ of the (second-moment) bulk correlation length $\xi$ - behaves as

$$
m \sim\left|\tau_{0}-\tau_{0 \mathrm{c}}\right|^{\nu} \quad \text { with } \quad \nu=\left(2+\eta_{\phi^{2}}^{*}\right)^{-1}
$$


Furthermore, integration of Eqs. (91) and (90) gives the asymptotic dependence

$$
Z_{\kappa} \sim m^{\eta_{\kappa}^{*}}, \quad \kappa=\phi, \phi^{2}, \lambda,(1, \infty),
$$

for $(u, f) \rightarrow\left(u^{*}, f^{*}\right)$ or $m \rightarrow 0$. We insert this result for $\kappa=\lambda$ into Eq. (87), substitute expression (93) for $\eta_{\lambda}^{*}$, and arrive at

$$
\lambda \sim m^{\mathfrak{z}} \lambda_{0},
$$

where $\mathfrak{z}$ is the dynamic bulk exponent (71). With the aid of these results, it is straightforward to deduce the scaling forms

$$
\begin{aligned}
& G_{\infty}^{(\tilde{N}, N ; \tilde{M}, M)}\left(\{\boldsymbol{x}\}, t ; \tau_{0}, u_{0}, f_{0}, \lambda_{0}\right) \\
& \approx m^{(\tilde{N}+N) \beta+(\tilde{M}+M) \beta_{1}^{\text {ord }}} \Xi_{\infty}^{(\tilde{N}, N ; \tilde{M}, M)}\left(\{m \boldsymbol{x}\}, \lambda_{0} t m^{\mathfrak{z}}\right) .
\end{aligned}
$$

Here $\beta=(\nu / 2)(d-2+\eta)$ is a standard bulk critical index, while $\beta_{1}^{\text {ord }}$ is its surface counterpart for the ordinary transition. (For recent estimates of the numerical value of the latter at $d=3$, see Ref. 26, its references, and Ref. 31.) The set $\{\boldsymbol{x}\}$ comprises all position coordinates on which the respective function depends. The case $(\tilde{N}, N ; \tilde{M}, M)=(0,0 ; 1,1)$ is special in that the term $\delta(t-\tilde{t}) \delta\left(\boldsymbol{x}_{\|}-\tilde{\boldsymbol{x}}_{\|}\right) \hat{G}^{(0,0 ; 1,1)}(\boldsymbol{p}=\mathbf{0}, \omega=0)$, which results from the subtraction in Eq. (99), should be subtracted on the left-hand side of Eq. (110). We have suppressed this term, because we consider $G^{(0,0 ; 1,1)}$ here not as a distribution, but as a function for $t-\tilde{t}>0$.

Let us choose $(\tilde{N}, N ; \tilde{M}, M)=(0,2 ; 0,0)$ in Eq. (110) and consider the case of the spinspin cumulant (3). If we set $\lambda_{0}=1$ for convenience, we obtain the scaling form given by Eq. (73) in the limit $m \rightarrow 0$.

The scaling form of the surface structure function (74) at the ordinary transition can be derived from the expansion of $G^{(0,0 ; 0,2)}$ in powers of $c_{0}^{-1}$ (see Eq. (110)). Alternatively, we can combine the CS equations (102) with Eq. (110) and the BOE (75) (applied to the renormalized theory) to conclude that the coefficient function $D(z, t)$ asymptotically satisfies the CS equation

$$
\left[\mathcal{D}_{m}-\frac{\eta_{1, \infty}}{2}\right] D(z, t)=0 .
$$

In the limit $m \rightarrow 0$, Eq. (111) yields a leading short-distance singularity of the form

$$
D(z, t) \underset{z \rightarrow 0}{\approx} D_{0} z^{1+\eta_{1, \infty}^{*} / 2},
$$


where the exponent $\eta_{1, \infty}^{*}$ can be expressed in terms of $\eta$ and the surface correlation index $\eta_{\|}^{\text {ord }}=2+\eta+\eta_{1, \infty}^{*}$. It follows that the scaling function $\Upsilon$ in Eq. (73) must behave as

$$
\Upsilon\left(\mathrm{z}, \mathrm{z}^{\prime} ; \mathrm{t}\right) \underset{\mathrm{z}, \mathrm{z}^{\prime} \rightarrow 0}{\approx}(\mathrm{zz})^{\left(\eta_{\|}^{\text {ord }}-\eta\right) / 2} \Upsilon_{0}(\mathrm{t})
$$

This in turn implies that the Fourier transformed surface structure function $\hat{C}_{11}(\boldsymbol{p}, \omega)$ at $T_{\mathrm{c}}$ can be written as

$$
\hat{C}_{11}(\boldsymbol{p}, \omega) \approx p^{\eta_{\|}^{\text {ord }}-1-\mathfrak{z}} \sigma\left(\omega p^{-\mathfrak{z}}\right)
$$

The limit $\boldsymbol{p} \rightarrow \mathbf{0}$ exists. By consistency, we must therefore have

$$
\hat{C}_{11}(\mathbf{0}, \omega)=\text { const } \omega^{-\left(\mathfrak{z}+1-\eta_{\|}^{\text {ord }}\right) / \mathfrak{z}} .
$$

In the next section, we will check the predictions (114) and (115) by means of accurate Monte-Carlo spin dynamics simulations.

\section{MONTE-CARLO SPIN DYNAMICS SIMULATION}

The Monte-Carlo spin dynamics simulation works as follows: A Monte-Carlo simulation

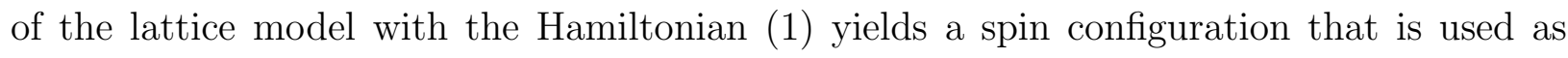
initial condition for the integration of the equations of motion (2). When the integration is completed, the time evolution of the spin configuration is analyzed for position and time displaced correlations. The correlation functions are then stored in arrays, and a new initial condition is generated by the Monte-Carlo simulation. Typically, this is repeated 700 to 1000 times. The correlation function $C^{\alpha \beta}\left(\boldsymbol{r} ; z, z^{\prime} ;\left|t-t^{\prime}\right|\right)$ [cf. Eq. (33)] is finally obtained by averaging over the individual measurements.

The Monte-Carlo algorithm is chosen as a hybrid scheme consisting of Metropolis sweeps, Wolff single cluster updates, 国 and overrelaxation sweeps. Carlo step involves 10 individual steps, each of which can be one of the updates listed above. Both the Metropolis and the Wolff algorithm work in the standard way, where the reduced coordination number of the lattice at the surfaces and the modified surface coupling $J_{1}$ must be taken into account. The acceptance probability $p$ of a proposed spin flip in the Metropolis algorithm is defined by $p(\Delta E)=1 /\left[\exp \left(\Delta E / k_{B} T\right)+1\right]$, where $k_{B}$ is Boltzmann's constant and $\Delta E$ is the change in configurational energy of the proposed move. 
The overrelaxation part of the algorithm performs a microcanonical update of the configuration by sequentially rotating each spin in the lattice such that its contribution to the energy of the whole configuration remains constant. The implementation of this update scheme is straightforward. To see this, note that the energy of a spin with respect to its neighborhood has the functional form of a scalar product according to Eq. (11). Therefore, a spin can be rotated about the direction of the local field generated by its neighbors without changing the local energy. The angle of rotation can be chosen randomly for each spin. However, in order to have minimal autocorrelation times, a reflection-i.e., a rotation of a spin by 180 degrees - turns out to be the most efficient overrelaxation move. In one overrelaxation sweep this update is applied in sequence to every spin of the lattice.

Typically, a hybrid Monte-Carlo step consists of two sweeps of the whole lattice via the Metropolis algorithm (M), four sweeps of the whole lattice by means of the overrelaxation algorithm (O) described above, and four single cluster updates according to the Wolff algorithm $(\mathrm{C})$. The individual updates are mixed automatically in the program so as to generate the update sequence M O C O C M O C O C . As our random number generator, we have utilized the shift-register generator R1279 defined by the recursion relation $X_{n}=X_{n-p} \oplus X_{n-q}$ for $(p, q)=(1279,1063)$. Generators like this one are among the fastest available. However, they are known to cause systematic errors in combination with the Wolff algorithm. 54 For lags $(p, q)$ as large as the ones used here, these errors are much smaller than typical statistical errors. The hybrid nature of the algorithm reduces them further.5

Using this Monte-Carlo scheme, we have investigated lattice sizes $L$ between $L=12$ and $L=72$. The integrated correlation time of the hybrid algorithm is determined by the autocorrelation function of the energy or, equivalently, by the autocorrelation function of the modulus of the magnetization. Either quantity is $O(3)$ symmetric, and for sufficiently long times, the decay of the corresponding autocorrelation functions is governed by the same autocorrelation time. This time scale characterizes the slowest mode of the Wolff algorithm, so it also determines the correlation time of our hybrid Monte-Carlo algorithm. Note that the autocorrelation time of the Metropolis algorithm is determined by the decay of the autocorrelation function of the order parameter, which decays particularly slowly near the critical point (critical slowing down). For the hybrid scheme described above, the autocorrelation time does not exceed 10 hybrid Monte-Carlo steps for the largest lattice size at $T=T_{\mathrm{c}}$. In order not to obtain too strongly correlated initial conditions for the equation 
of motion, an initial condition is generated every tenth hybrid Monte-Carlo step.

The integration procedure for the equations of motion is completely separated from the Monte-Carlo part of the program. The second-order sublattice decomposition integrator described in Ref. 29 is used here. Long-time stability is provided by the exact conservation of energy [see Eq. (2)] and spin normalization. The magnetization is only conserved within the accuracy of the discretization, i.e., to second order in the time step. Typical time steps $\delta t$ used here range from $\delta t=0.04 / J$ to $\delta t=0.08 / J$, depending on the size of the system. For the largest system $(L=72)$, the total integration time is $\tau_{I}=8192 / J$; in this case $\delta t=0.08 / J$ was used. Note that the decomposition integrator $I(\delta t)$ has the exact time inversion property $I(-\delta t)=I^{-1}(\delta t)$. This guarantees that the time evolution of discretization errors, such as those affecting the conservation of the magnetization, does not contain systematic drifts.29

If the algorithm is implemented on a single processor machine, the major part of the CPU time is consumed by the integration of the equations of motion. This fraction increases with system size because the CPU time needed for the integration scales as $\tau_{I} L^{3}$, whereas that of a hybrid Monte-Carlo step scales as $L^{3}$. If Wolff updates are used exclusively, the average scaling is reduced to $L^{2-\eta} \underline{52}$ For the purposes of the present investigation the integration time $\tau_{I}$ has to be chosen such that the slowest spin wave or spin diffusion modes in the system can be identified. At $T=T_{\mathrm{c}}$ this means that $\tau_{I} \sim L^{\mathfrak{z}}$ [see Eq. (71)]. Below $T_{\mathrm{c}}$, one must have $\tau_{I} \sim L^{2}$ for an isotropic ferromagnet in order to resolve the slowest spin-wave mode. Above $T_{\mathrm{c}}$, the dynamics is dominated by spin diffusion, which also requires $\tau_{I} \sim L^{2}$ for the resolution of the slowest modes. It is therefore very desirable to distribute the integration task of the simulation over several processors on a parallel machine.

A simple and very efficient implementation on a parallel machine with at least four processors can be constructed according to the following master-slave idea: The master process runs the Monte-Carlo part of the simulation to provide initial conditions on demand, and the slave processes integrate the equations of motion for different initial conditions in parallel and analyze the correlations. The amount of communication among the processes is determined by the transfer of initial configurations from the master to the slaves at the beginning of the simulation, and by the transfer of the correlation data from the slaves to the master for the final evaluation and output. If $N$ processors in parallel are used in this way, the speedup is very close to the theoretical limit $N-1$ for sufficiently large 
integration times $\tau_{I}$. We have implemented the master-slave version of the spin dynamics algorithm on the Alpha Linux cluster ALiCE at the Institut für angewandte Informatik at the Bergische Universität Wuppertal, using up to 32 processors in parallel for the largest systems. Communication between the processors is facilitated by the MPI (message passing interface) communication library.

A well-known major problem one is faced with in any computer simulation study of critical behavior is how to extract the asymptotic critical behavior from the data. This is particularly challenging in our case since we have to cope with two additional complications: surface critical behavior and dynamics. In the asymptotic critical regime the value of the ratio $r_{1} \equiv J_{1} / J$ does not matter if $d=3$ because surfaces of three-dimensional isotropic Heisenberg ferromagnets are always disordered in the absence of external fields. Thus such systems always belong to the ordinary surface universality class. However, to what extent the asymptotic scaling can actually be observed in a computer simulation on finite systems is a completely different issue.

The experience made in a previous study of the static case by one of us 31 suggests that it should be possible to avoid extended crossover regimes by a careful choice of the ratio $r_{1}$. In order to find out which value of $r_{1}$ is optimal in the sense of giving the largest asymptotic regime, we proceed as in Ref. 31: We consider the magnetization profile in thermal equilibrium, determine $r_{1}$ in such a way that a discrete version of the Dirichlet boundary condition holds, and then verify that this choice suppresses corrections to the asymptotic behavior, making the asymptotic regime larger than for alternative values of $r_{1}$. Let us explain this in detail. The equilibrium profile is

$$
m(z) \equiv\left\langle\frac{\boldsymbol{M}_{\mathrm{tot}}}{\left|\boldsymbol{M}_{\mathrm{tot}}\right|} \cdot \sum_{i_{1}, i_{2}=0}^{L-1} \boldsymbol{S}_{\left(i_{1}, i_{2}, i_{3}\right)}\right\rangle,
$$

where $\boldsymbol{M}_{\text {tot }} \equiv \sum_{\boldsymbol{i}} \boldsymbol{S}_{\boldsymbol{i}}$ is the total magnetization, while $z \equiv i_{3}+1 / 2$ with $i_{3}=0, \ldots, L-1$ indicates a lattice plane parallel to the surfaces [cf. Eq. (11)]. Note that according to this definition of $z$, the 'boundary planes' $z=1 / 2$ and $z=L-1 / 2$ of the system are located half a lattice constant away from the first and last lattice layers, respectively. With this convention, the lattice model defined by Eq. (11) may be viewed as a discrete version of the Ginzburg-Landau Hamiltonian (6), where the order parameter of the numerical cell $\boldsymbol{i}$ is represented by the spin $\boldsymbol{S}_{\boldsymbol{i}}$ at its center.

The bulk magnetization $m_{\mathrm{b}}$ can be approximated by the value of the magnetization in the 
center layer of the system, i.e., $m_{\mathrm{b}} \equiv m\left(z_{\text {mid }}\right)$ with $z_{\text {mid }}=L / 2$ when the number of layers, $L$, is odd. For even $L, z_{\text {mid,1 }}=(L-1) / 2$ and $z_{\text {mid,2 }}=(L+1) / 2$ are equivalent choices for $z_{\text {mid }}$; in this case, $m_{\mathrm{b}} \equiv\left(m\left(z_{\text {mid,1 }}\right)+m\left(z_{\text {mid,2 }}\right)\right) / 2$ is interpreted as the bulk magnetization. The ratio $m(z) / m_{\mathrm{b}}$ then depends only on $z / L$, which motivates us to define the scaling function

$$
M(z / L) \equiv m(z) / m_{\mathrm{b}}
$$

where $T=T_{\mathrm{c}}$ is assumed. The analysis of the data reveals that the scaling function $M(\zeta \equiv$ $z / L)$ can be represented by the simple fit formula

$$
M(\zeta)=B_{M}\left[\left(\zeta+\zeta_{0}\right)\left(1-\zeta+\zeta_{0}\right)\right]^{\left(\beta_{1}-\beta\right) / \nu}
$$

to a remarkable accuracy. Here $\zeta_{0}=z_{0} / L$ is the scaled extrapolation parameter. In analyzing the data we have accepted the estimates $\beta=0.3662 \pm 0.0025$ and $\nu=0.7073 \pm 0.0035$ of Ref. 56, and utilized the value $\beta_{1}=0.834(6)$ of Ref. 31 .

From a least square fit of Eq. (118) to the data for various system sizes we obtain the extrapolation parameter $z_{0}$ in units of the lattice spacing. For the choices $J_{1} / J=0.3$ and 1.0, we find $z_{0} \simeq-0.34$ and $z_{0} \simeq 0.46$, respectively. On the other hand, $z_{0}$ vanishes within the statistical errors if $J_{1} / J=0.73 .31$ To put these findings in perspective, some explanations are necessary. Owing to our definition of $z$ [given below Eq. (116)], a fit curve (118) with scaled extrapolation parameter $\zeta_{0}=0$ means that the measured magnetization profile extrapolates to zero half a lattice constant away from the outermost layers $i_{3}=0$ and $i_{3}=L-1$ of our lattice model. In this sense, the profile satisfies a Dirichlet boundary condition on the scale of the lattice constant in this special case.

Let us emphasize that such a boundary condition on a microscopic scale must not be confused with the Dirichlet boundary condition which the order parameter satisfies at the ordinary transition on long scales, irrespective of the precise value of the ratio $r_{1} \equiv J_{1} / J$. The latter is an asymptotic long-scale property, associated with the corresponding ordinary fixed point of the RG, and hence universal. By contrast, the boundary condition that the order-parameter profile of a given microscopic model is found to satisfy on microscopic scales generally depends on microscopic details, and is therefore a nonuniversal property (cf. the discussion in Sec. III.C.9 of Ref. 5).

On the level of a mesoscopic description via our continuum model, a Dirichlet boundary condition can be enforced on the mesoscopic length scales on which such a description 
makes sense (several lattice constants) by setting the enhancement variable $c_{0}$ to the fixedpoint value $+\infty$. For values $c_{0}<\infty$, the Dirichlet boundary condition does not hold on mesoscopic scales, neither for the regularized nor for the renormalized theory. In other words, a $c_{0}$-dependent extrapolation parameter $z_{0} \neq 0$ occurs. This deviation from the Dirichlet boundary condition corresponds to a correction to scaling: It is irrelevant inasmuch as it

vanishes in the limit $z_{0} / z \rightarrow 0$. Choosing a particular value $r_{1}=r_{1}^{(\mathrm{D})}$ for the ratio of interaction constants such that $z_{0} \simeq 0$ is an appealing way to mimic the Dirichlet boundary condition of the $c_{0}=\infty$ continuum theory on a lattice. As we know already for the static case from Ref. 31, and will verify for the dynamic theory below, this choice of $r_{1}$ suppresses corrections to scaling and hence enlarges the regime in which the asymptotic scaling behavior is observed.

The optimal value which yields a vanishing extrapolation parameter $z_{0}$ for temperatures sufficiently close to $T_{\mathrm{c}}$ and moderately large lattice dimensions $L$ is $r_{1}^{(D)}=0.73$. For values close to this optimal one, we have

$$
z_{0}=a\left(r_{1}-r_{1}^{(D)}\right)+\mathcal{O}\left[\left(r_{1}-r_{1}^{(D)}\right)^{2}\right]
$$

where $a$ is a factor of order unity. This behavior was already obtained in Ref. 31, where crossover effects and the behavior of the order parameter profile as a function of $r_{1}$ were investigated in more detail for the static case. The present work confirms these findings: Our results for the dynamic surface structure function presented in the next section are fully consistent with them.

\section{THE DYNAMIC SURFACE STRUCTURE FUNCTION}

Our simulation results are displayed in Figs. 1 14 They were obtained for $T=T_{\mathrm{c}}$, $L=72$, and the total integration time $\tau_{I}=8192 / J$. For the smallest accessible frequency $\omega_{\min }=2 \pi / \tau_{I}$, finite-size effects turned out to be negligible.

In Fig. 11 the structure function $\hat{C}_{11}(\mathbf{0}, \omega)$ is shown for different values of $r_{1}$ in comparison with Eq. (115). The exponent $\left(\mathfrak{z}+1-\eta_{\|}^{\text {ord }}\right) / \mathfrak{z}$ has the value $0.856 \pm 0.005$ that follows from the estimate $\mathfrak{z}(d=3)=2.482 \pm 0.002$ obtained by the substitution $\eta(d=3)=0.036 \pm 0.004 \sqrt[56]{ }$

in Eq. (71) and the current estimate of the surface correlation exponent $\eta_{\|}^{\text {ord }}(d=3)=1.358 \pm$ 0.01231 of the ordinary transition. 


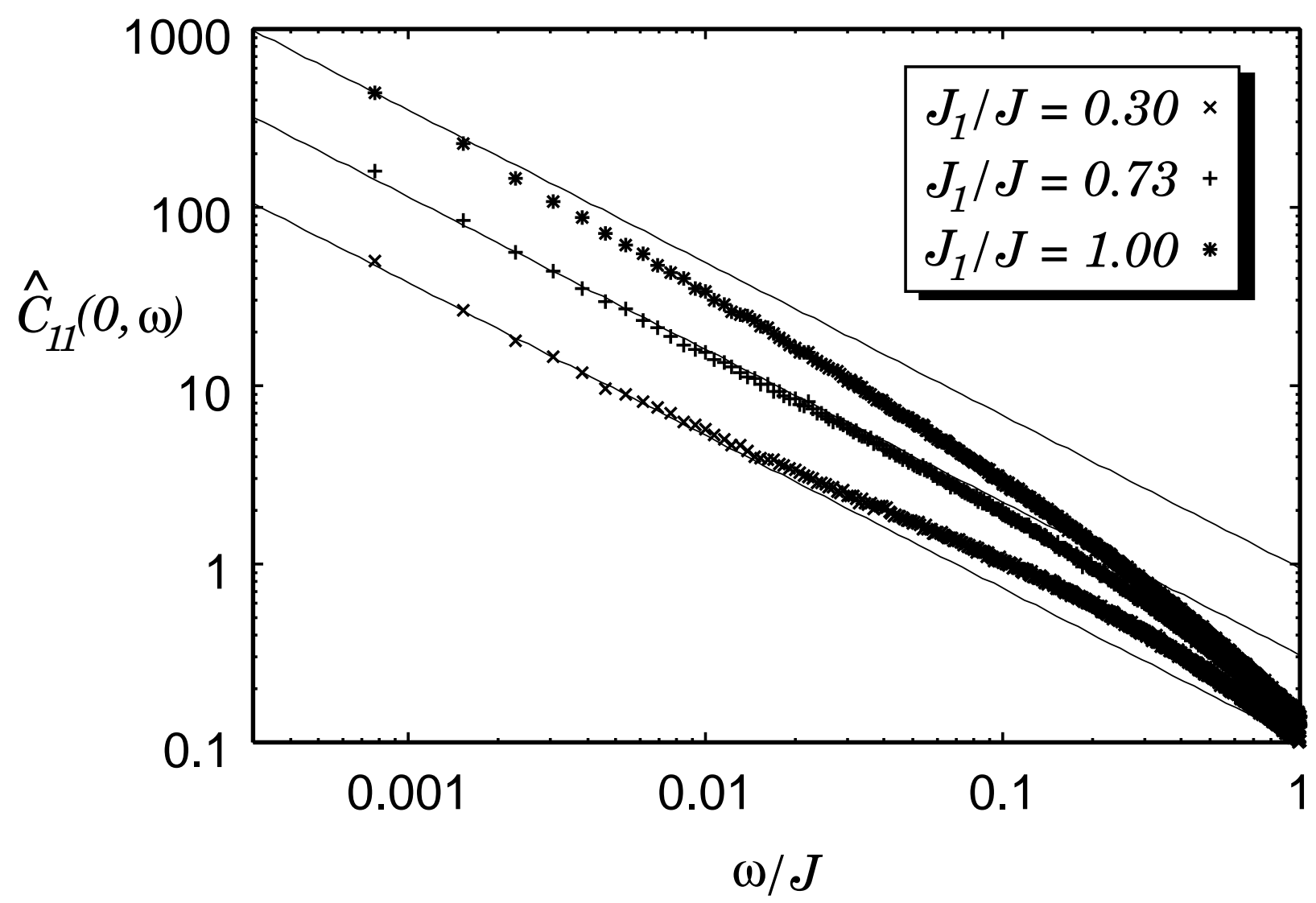

FIG. 1: Structure function $\hat{C}_{11}(\mathbf{0}, \omega)$ for $r_{1} \equiv J_{1} / J=0.3(\times), 0.73(+)$, and $1.0(*)$. Error bars (one standard deviation) are smaller than the symbol sizes. The solid lines indicate the theoretically expected power law (115) for $\omega \rightarrow 0$.

The dependence of $\hat{C}_{11}(\mathbf{0}, \omega)$ on $r_{1}$ is particularly interesting. If $r_{1}$ is small $\left(r_{1}=0.3, \times\right)$, our simulation data approach the asymptotic power law (115) from above, whereas for larger values of $r_{1}\left(r_{1}=1, *\right)$, the asymptotic power law is approached from below. In the latter case, the data even remain outside the asymptotic regime for the frequency range shown in Fig. 1. The best agreement with Eq. (115) over the largest frequency range is obtained for the choice $r_{1}=0.73(+)$, which has already been identified as optimal in the sense that the extrapolation parameter $z_{0}$ for the magnetization profile vanishes (see Eq. (119)). The deviations from the power law (115) for $r_{1} \neq 0.73$ can apparently be attributed to dynamic surface-induced corrections to the asymptotic behavior that originate from the nonzero, $r_{1}$-dependent value of the extrapolation parameter $z_{0}$.

Fig. 2 shows a scaling plot of $\hat{C}_{11}(\boldsymbol{p}, \omega)$, where $\boldsymbol{p}=\left(\frac{n \pi}{36}, 0,0\right)$ is oriented along the surface. As one sees, the scaling regime in $x$ shrinks as the mode index $n$ is increased from $1(\times)$ to 


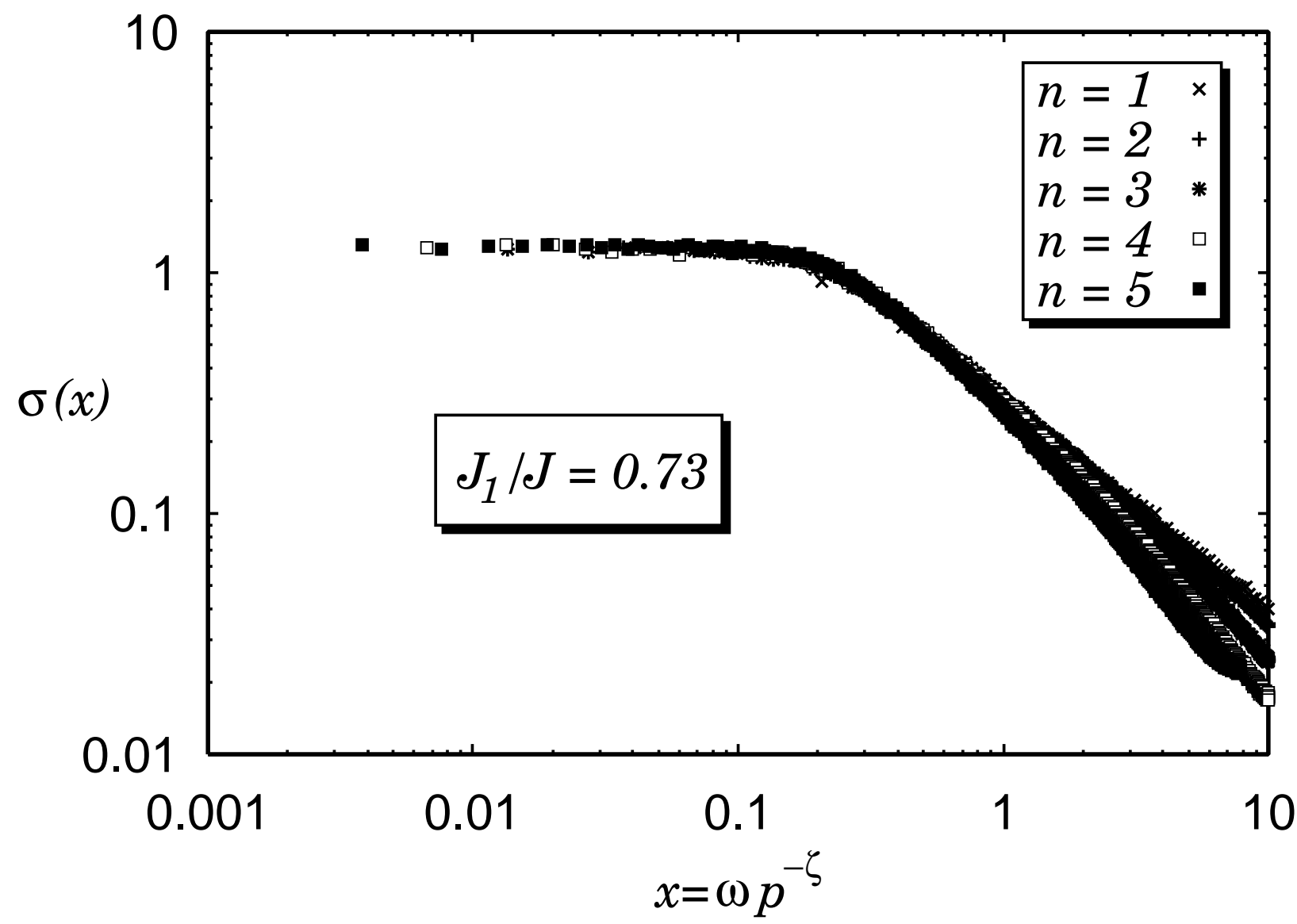

FIG. 2: Scaling function $\sigma(x)$ according to (114). Data obtained for $r_{1} \equiv J_{1} / J=0.73$ and $\boldsymbol{p}=\left(\frac{n \pi}{36}, 0,0\right)$, with $n=1, \ldots, 5$, are shown. Error bars (one standard deviation) are smaller than the symbol sizes. The data follow Eq. (120) up to $x \simeq 1$. The data for $x \geq 1$ are outside the scaling regime.

$5(\boldsymbol{\square})$. For $x<1$, the shape of the scaling function in Eq. (114) is described surprisingly well by the fit function 18

$$
\sigma(x)=\sigma_{0}\left[1+\left(x / x_{0}\right)^{4}\right]^{\left(\eta_{\|}^{\text {ord }}-\mathfrak{z}-1\right) / 4 \mathfrak{z}},
$$

which is inspired by the known zero-loop result. 여요 The exponent at the square bracket is chosen so as to reproduce Eq. (115) in the limit $x \rightarrow \infty(p \rightarrow 0$ at fixed $\omega \neq 0)$. The amplitude $\sigma_{0}$ and the crossover parameter $x_{0}$ are used as fit parameters.

The agreement between the data displayed in Figs. 1 and 2 and the scaling forms (114) and (115) is quite satisfactory. However, on closer inspection small deviations are found to remain. Note that as pointed out at the end of the previous section and in analogy with the results of Ref. 31 for the equilibrium case, the choice $r_{1}=0.73$ yields indeed the largest 
regime in which asymptotic scaling holds (see Fig. 1).

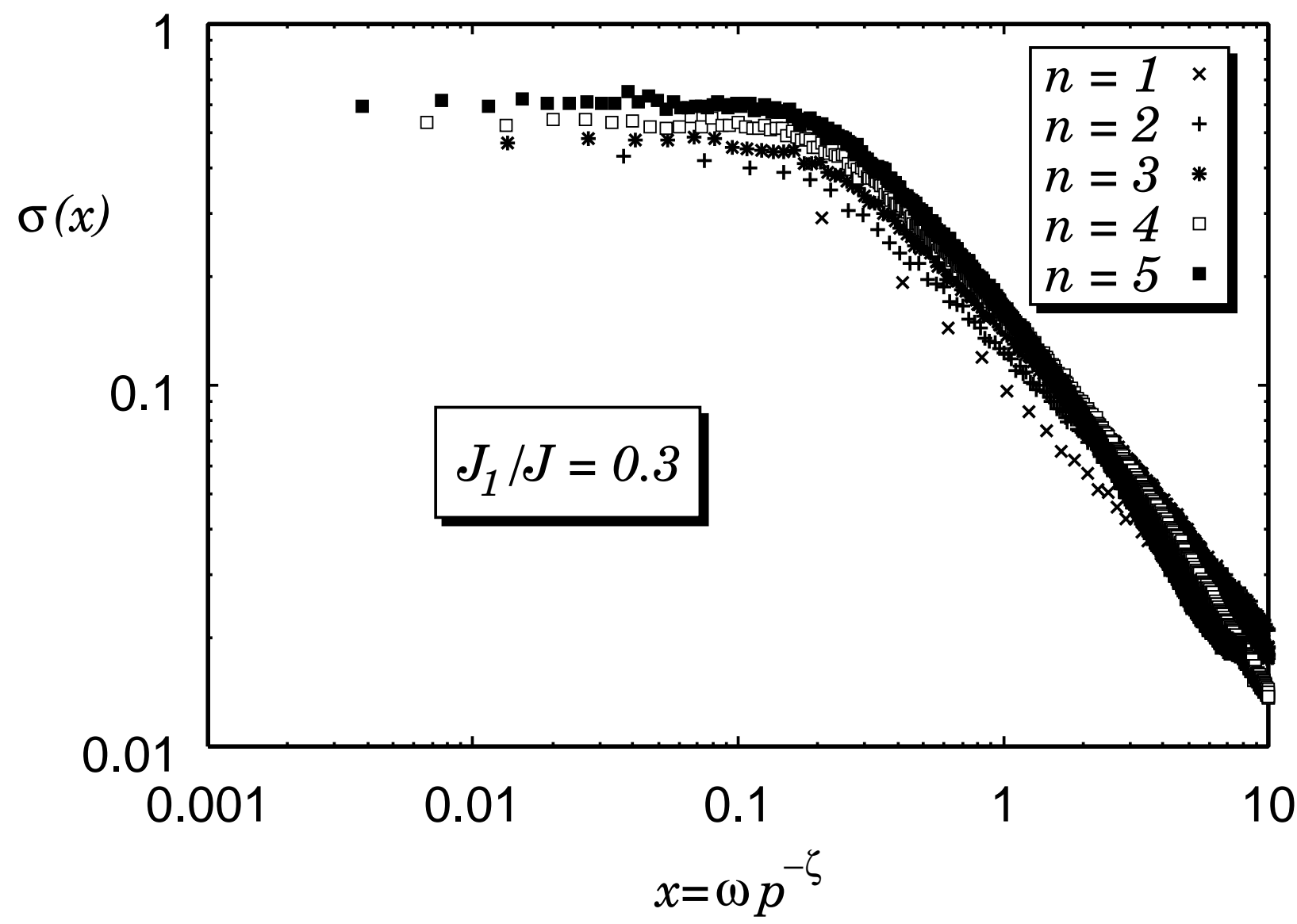

FIG. 3: Scaling plot of the surface structure function for $r_{1} \equiv J_{1} / J=0.3$ and $\boldsymbol{p}=\left(\frac{n \pi}{36}, 0,0\right)$, with $n=1, \ldots, 5$. Error bars (one standard deviation) are smaller than the symbol sizes. The data do not follow Eq. (120).

Hence we expect the choice $r_{1}=0.73$ to be optimal also for the surface structure factor at finite momentum transfer $p$. Our results for $r_{1}=0.3$ and $r_{1}=1.0$ depicted in Figs. 3 and 4 confirm this expectation. Figure 3 shows that the data for $r_{1}=0.3$ approach the scaling function $\sigma(x)$ of Fig. 2 monotonically from below as the mode number $n$ is increased from 1 to 5. The nonasymptotic surface-induced corrections are so large that the data for different $n$ (i.e., momentum transfers $\mathbf{p}$ ) are well separated even on a logarithmic scale. In other words, no data collapse nearly as nice as in Fig. 2 occurs, although the crossover parameter $x_{0}$ appears to be consistent with the results displayed there.

Our results for $r_{1}=1.0$ (see Fig. 价) show a similar behavior, except that the scaling function $\sigma(x)$ now is monotonically approached from above as the mode number $n$ increases. 
The crossover parameter $x_{0}$ is again consistent with our findings in Figs. 2 and 3 . The nonasymptotic surface-induced corrections are as large as in Fig. 3 but have opposite sign.

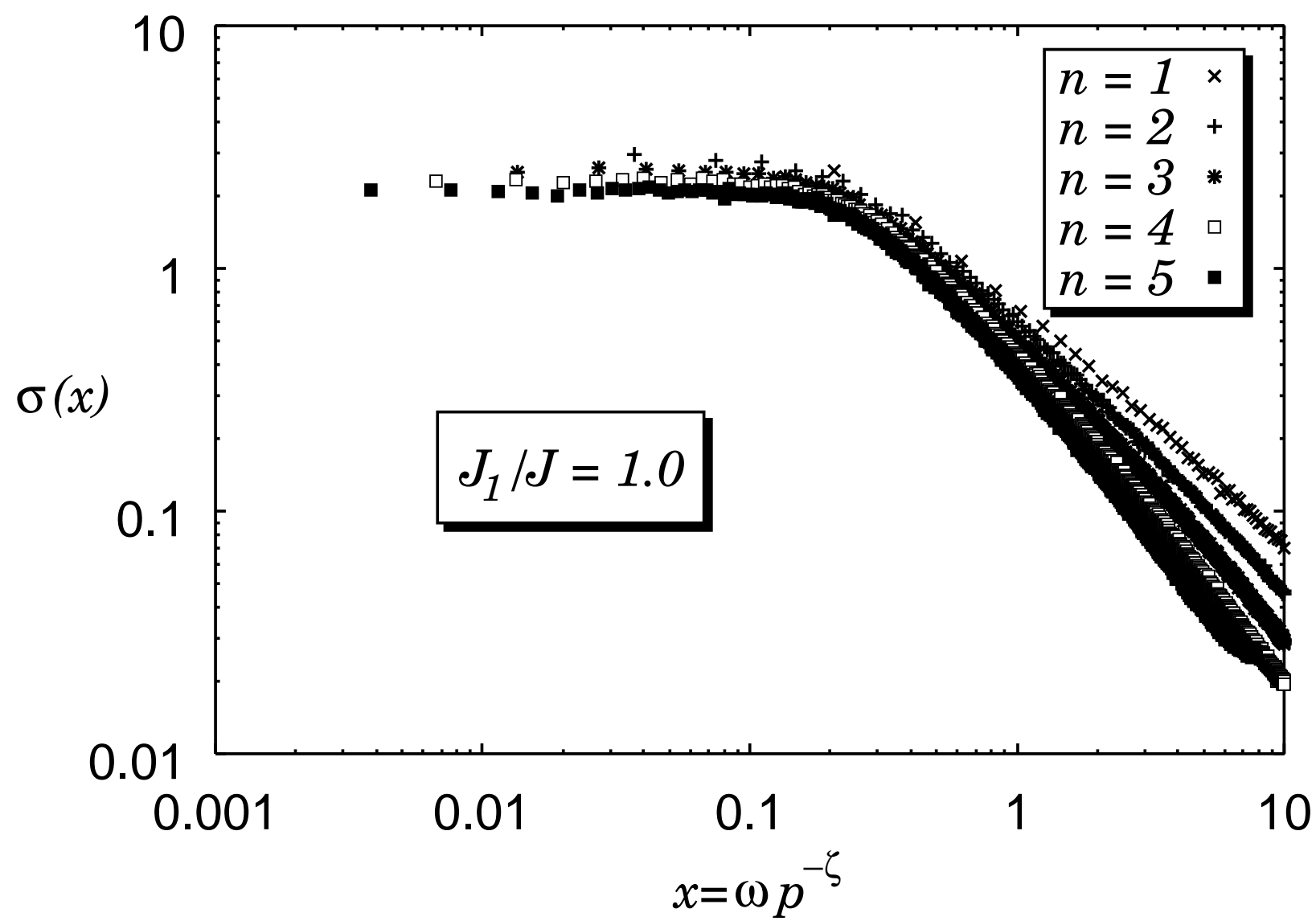

FIG. 4: Scaling plot of the surface structure function for $r_{1}=1.0$ and $\boldsymbol{p}=\left(\frac{n \pi}{36}, 0,0\right)$, with $n=$ $1, \ldots, 5$. Error bars (one standard deviation) are smaller than the symbol sizes. The data do not follow Eq. (120).

From these results a consistent numerical picture of dynamic surface scaling emerges. For the optimal choice $r_{1}=0.73$, our simulation data for the dynamic structure function bear out quite convincingly the asymptotic behavior we predicted on the basis of our RG work. For values of $r_{1}$ deviating significantly from 0.73 , the data exhibit pronounced corrections to scaling. These entail that our data for values like $r_{1}=1$ and 0.3 do not exhibit directly the asymptotic scaling form and power law of the surface structure function $\hat{C}_{11}(\boldsymbol{p}, \omega)$ for nonzero and zero parallel momentum $\boldsymbol{p}$, respectively. Yet they seem to be consistent both with the theoretically predicted asymptotic behavior as well as with the one extracted from our simulation data for $r_{1}=0.73$ because the observed deviations appear to be attributable to corrections to scaling. 
In order to demonstrate universality, it would clearly be very desirable to verify the approach to the asymptotic critical behavior also for unfavorable values of $r_{1}$ like 1.0 and 0.3. One conceivable way of trying to reach this goal is by means of brute-force numerical means. However, in view of the enormous numerical effort that was necessary to produce the above simulation results, we do not consider this to be promising strategy at present.

We believe that a better strategy would be the incorporation of nonasymptotic correction terms in the analysis of the simulation data. Unfortunately, there are various sources of such corrections, and detailed knowledge about their form and strength is either scarce or not available. A systematic investigation of the various kinds of nonasymptotic corrections of static and dynamic origin that might play a role in the analysis of the surface critical behavior we are concerned with here evidently requires more numerical and analytical work, and is beyond the scope of this paper. Let us therefore simply discuss some possible sources of deviations from scaling, beginning with the ones that do not correspond to corrections to scaling.

Two obvious sources of this latter kind are insufficient momentum and frequency resolution. By virtue of the relation $\delta p=2 \pi / L$ the momentum resolution $\delta p$ is intimately linked to the system size $L$, which despite formidable progress in simulation techniques and computational resources still is a serious limiting factor. The frequency resolution $\delta \omega=2 \pi / \tau_{I}$ is limited by the total integration time $\tau_{I}$. From our data for $C_{11}(\boldsymbol{p}, t)$ (not shown) we conclude that $\tau_{I}$ is sufficiently long. The frequency resolution $\delta \omega / J \simeq 7.7 \times 10^{-4}$ that is available here rivals that of neutron scattering experiments. 3 The momentum resolution is given by $\delta p \simeq 0.087$ in units of the inverse lattice constant for our largest systems, and is therefore much more restrictive.

One familiar type of corrections to scaling are those induced by deviations of the coupling constant $u$ from its fixed point value $u^{*}$. Just as in the static case, they are governed by the Wegner exponent $\omega_{u} \equiv \beta_{u}^{\prime}\left(u^{*}\right)$ whose value is $\simeq 0.8$ in $d=3$ dimensions. 6 analogous corrections to scaling result from the RG flow of the mode-coupling interaction constant $f$. Upon linearizing the flow about the infrared-stable fixed point $\left(u^{*}, f^{*}\right)$, one obtains in addition to $\omega_{u}$ a second correction-to-scaling exponent, $\omega_{f} \equiv \beta_{f}^{\prime}\left(u^{*}, f^{*}\right)$, which in contrast to the former is of purely dynamic origin. We are not aware of any reliable estimates of $\omega_{f}$ for $d=3$ 国

Other potentially dangerous corrections might be caused by a previously mentioned im- 
portant difference of the dynamics of the simulated lattice model and the semi-infinite continuum model $\mathrm{J}$ : that the former conserves the energy while the latter does not. In Appendix A we generalize model $J$ for the bulk case by incorporating energy conservation. The resulting model is analogous to model $\mathrm{D}$ (with $n=3$ components), and reduces to this when the mode-coupling interaction constant $f$ is set to zero. We show that the ratio $\lambda / \lambda^{(\mathcal{E})}$ of transport coefficients (where $\lambda^{(\mathcal{E})}$ denotes the analog of $\lambda$ for the energy density $\mathcal{E}$ ) transforms under the RG as $\ell^{\mathfrak{z}-2}$ in the long length-scale limit $\ell \rightarrow 0$. Since $\mathfrak{z}-2=(d-2+\eta) / 2$, which is $\simeq 0.5$ in three dimensions, the ratio approaches indeed zero, albeit with a considerably smaller power than in the case of model $\mathrm{D}$ (where the value of this exponent is $2-\eta \simeq 2$ ).

Hence two conclusions may be drawn: First, in order to obtain the asymptotic critical behavior of order parameter cumulants we can take the limit $\lambda / \lambda^{(\mathcal{E})} \rightarrow 0$. If one sets $1 / \lambda^{(\mathcal{E})}=$ 0 from the outset, the energy density relaxes instantaneously, This means that the effects produced by the coupling to the energy density correspond to a change of the parameters of the original model J, up to corrections due to irrelevant operators. In other words, the energy conservation should not affect the asymptotic critical behavior, so that our lattice model should belong to the universality class of our semi-infinite model J. Second, we cannot rule out that the corrections to the asymptotic behavior induced by the conservation of the energy are less important for an improved analysis of the numerical data presented above than the previously mentioned corrections to scaling. To assess the relative importance of the various types of corrections to scaling seems difficult without reliable additional information based on detailed calculations.

The corrections to scaling we have just considered are associated with irrelevant bulk variables and hence are not specific to systems with surfaces. Analogous ones are induced by irrelevant surface variables. A well-known example are the corrections $\sim \ell$ resulting from deviations of $1 / c_{0}$ (the reciprocal surface enhancement variable) from the fixed-point value $1 / c_{\text {ord }}^{*}=0$. One evident consequence of such corrections (which is, however, not the only one when Landau theory is not valid) is that the Dirichlet boundary condition the orderparameter density satisfies at the ordinary fixed point ceases to hold. In view of the two observations made above - namely, (i) that the choice $r_{1}=0.73$ suppresses corrections to scaling both in the case of the dynamic structure functions as well as in static quantities, 31

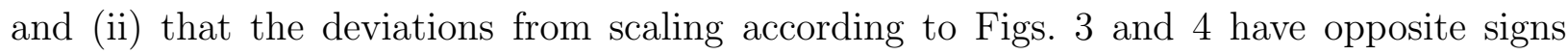
depending on whether $r_{1}$ is bigger or smaller than the optimal value 0.73 - the corrections 
to scaling which the finiteness of $c_{0}$ induces appear to play a major role.

\section{SUMMARY AND CONCLUSIONS}

We have presented a detailed study of the surface critical behavior of isotropic Heisenberg ferromagnets at the ordinary transition, using both sophisticated analytical tools as well as high-precision Monte-Carlo spin dynamics simulations. In the analytical part of our work a continuum model that represents the corresponding universality class of bulk and surface critical behavior-namely, an appropriate semi-infinite extension of model J-has been formulated and its field theory constructed. To this end we have determined the relevant boundary contributions to the dynamic action functional which are compatible with the general features (symmetries, detailed balance, locality assumptions, conservation laws, etc.) of this class of systems. These were shown to correspond to boundary conditions for the resulting dynamic field theory.

In order to investigate the critical behavior of this semi-infinite model $\mathrm{J}$, we have employed two distinct RG schemes. The first is a massless one based on the expansion about the upper critical dimension $d_{\mathrm{J}}^{*}=6$. To avoid the difficulties it has in handling the problem adequately below the upper critical dimension $d^{*}=4$ of the static theory, we have designed and utilized an appropriate extension of the massive RG scheme for bounded systems of

Diehl and Shpot.25,26 As usual, this works in fixed dimensions, and avoids the dimensionality expansion. By combining the resulting RG equations with the boundary operator expansion (75), we have been able to obtain detailed predictions for the scaling behavior of the surface structure function (5). The involved critical exponents which govern power laws like (115) are related to known static bulk and surface critical exponents. In particular, there is no independent new dynamic exponent associated with the surface.58

We have checked our predictions by means of extensive Monte-Carlo spin dynamics simulations. Our results depicted in Figs. 1 and 2 corroborate the predicted dynamic scaling behavior. In order to obtain such a good manifestation of scaling, we have found it helpful and necessary to choose the ratio $r_{1}=J_{1} / J$ of the surface and bulk exchange integrals $J_{1}$ and $J$ such that corrections to scaling are suppressed. To achieve this objective we have optimized the value of $r_{1}$ by requiring that the Monte Carlo data yield an equilibrium orderparameter profile which satisfies a Dirichlet boundary condition on the scale of the lattice 
constant in the sense described in Sec. [V.

According to our results displayed in Figs. 1 4 , this procedure is quite effective: For $r_{1}=0.73$ and values inside a narrow range around this optimal one, the simulation data for the dynamic structure functions exhibit clear evidence of dynamic scaling in conformity with our predictions. On the other hand, for values of $r_{1}$ outside this regime, the data collapse is poor and the asymptotic behavior cannot be identified in a convincing fashion. These

observations are in conformity with those made in a previous Monte Carlo investigation of the static surface critical behavior by one of us 31 However, in the study of static quantities one is in a much better position because the scaling regimes can be reached reasonably well even for non-optimal values of $r_{1}$. This fact lends support to our belief that the dynamic scaling behavior seen for $r_{1}=0.73$ can be trusted.

Finally, let us note that we have not taken into account any dipolar forces in our study. To check our result by experiments (as should become feasible in the near future thanks to facilities like the X-ray free electron laser 17 ), one must choose systems for which such forces are negligible. Since even weak dipolar forces lead to the formation of domains, one must also make sure that single domains are investigated.

\section{Acknowledgments}

We are grateful to $\mathrm{K}$. Wiese for discussions during the beginning phase of this work. M.K. is indebted to the Institut für angewandte Informatik at the Bergische Universität Wuppertal for providing access to the parallel cluster ALiCE. Partial support by DFG (for M.K. via the Heisenberg program under grant \# Kr 1322/2-1, for H.K. and H.W.D. via the Leibniz program Di 378/2-1 and SFB 237) is gratefully acknowledged.

\section{APPENDIX A: THE IRRELEVANCE OF ENERGY CONSERVATION}

The dynamics of the lattice model we simulated, defined via the equations of motion (2), conserves the energy. Our aim here is to show that this feature does not change the universality class, which should therefore be represented by the semi-infinite model J em-

ployed in our RG study. For the sake of simplicity, we restrict ourselves to demonstrating the irrelevance of energy conservation for the bulk case. The extension to the semi-infinite 
case should be obvious.

Conservation of energy means that the energy density $\mathcal{E}(\boldsymbol{x}, t)$ is a conserved density and hence a slow variable which should be retained in a coarse-grained description on mesoscopic time scales. Now, for vanishing mode-coupling constant $f_{0}$, model $\mathrm{J}$ reduces to model B. How to modify the latter to account for energy conservation is well known and leads to model D. 3 We can adapt the dynamics of model J along similar lines. The obvious result is a modification of model $\mathrm{J}$ that differs from model $\mathrm{D}$ through the addition of the former's mode-coupling terms. The Langevin equations of this two-density model, which we call J', read

$$
\dot{\phi}(\boldsymbol{x}, t)=\lambda_{0}\left(\triangle \frac{\delta \mathcal{H}^{\prime}}{\delta \boldsymbol{\phi}}+f_{0} \frac{\delta \mathcal{H}^{\prime}}{\delta \boldsymbol{\phi}} \times \boldsymbol{\phi}\right)+\boldsymbol{\zeta}(\boldsymbol{x}, t)
$$

and

$$
\dot{\mathcal{E}}(\boldsymbol{x}, t)=\lambda_{0}^{(\mathcal{E})} \triangle \frac{\delta \mathcal{H}^{\prime}}{\delta \mathcal{E}}+\vartheta(\boldsymbol{x}, t)
$$

where

$$
\mathcal{H}^{\prime}=\int_{\mathbb{R}^{d}}\left[\frac{1}{2}(\boldsymbol{\nabla} \phi)^{2}+\frac{\tau_{0}}{2} \phi^{2}+\frac{u_{0}}{4 !}|\boldsymbol{\phi}|^{4}+\frac{1}{2} \mathcal{E}^{2}+\frac{\gamma_{0}}{2} \mathcal{E} \phi^{2}\right]
$$

is the familiar Hamiltonian employed in the definition of models C, D, and $\mathrm{E}$ (for the here considered case of an $n=3$ component order parameter $\phi$ ). Both $\boldsymbol{\zeta}$ as well as $\vartheta$ are Gaussian random forces with zero average; their variances are given by Eq. (8) and

$$
\left\langle\vartheta(\boldsymbol{x}, t) \vartheta\left(\boldsymbol{x}^{\prime}, t^{\prime}\right)\right\rangle=-2 \lambda_{0}^{(\mathcal{E})} \triangle \delta\left(\boldsymbol{x}-\boldsymbol{x}^{\prime}\right) \delta\left(t-t^{\prime}\right)
$$

respectively.

In the absence of coupling between the order parameter $\phi$ and the energy density $\mathcal{E}$, i.e., for $\gamma_{0}=0$, the dynamic exponent of $\mathcal{E}$ takes its Gaussian value

$$
\mathfrak{z} \mathcal{E}=2,
$$

corresponding to ordinary diffusion. It is not difficult to see that this result remains valid for $\gamma_{0} \neq 0$. For $d>4$, this follows immediately from the observation that $\gamma_{0}$ is irrelevant in the $R G$ sense.

In studying the more interesting case $d \leq 4$, we can benefit from well-known results for the static theory described by the Hamiltonian (A3), which is equivalent to the $\phi^{4}$ Hamiltonian (6) except for a change $u_{0} \rightarrow U_{0} \equiv u_{0}-3 \gamma_{0}^{2}$ of the interaction constant (see, 
for example, Refs. 59 and Ref. 3, and their references). As a consequence, the corresponding static renormalization functions can be expressed in terms of those of the $\phi^{4}$-theory. In particular, the renormalized analog $U$ of $U_{0}$ can be introduced in analogy to Eq. (79) via $U_{0}=Z_{u}(U) m^{4-d} U$, where $Z_{u}(u)$ is the renormalization function of Sec. IIID3. Likewise, the renormalization factor $Z_{\gamma}\left(U, \gamma^{2}\right)$ which relates $\gamma_{0}=m^{(4-d) / 2} Z_{\gamma} \gamma$ to its renormalized counterpart $\gamma$ can be expressed in terms of known renormalization functions of the $\phi^{4}$ theory. (It is a product of $Z_{\phi^{2}}(U)$ and a factor linear in $\gamma^{2}$ whose $U$-dependent coefficient derives from the additive counterterm that the $\phi^{4}$-vertex function $\Gamma_{\phi^{2} \phi^{2}}$ requires.59)

The resulting RG flow of $U$ and $\gamma$ has two nontrivial fixed points at $U=u^{*}$ : one at $\gamma^{*}=0$, and another one at $\left(\gamma^{*}\right)^{2}=$ const $\alpha / \nu$. The slopes $\partial \beta_{\gamma^{2}}\left(U=u^{*}, \gamma^{2}\right) / \partial \gamma^{2}$ of the beta function $\left.\beta_{\gamma^{2}} \equiv m \partial_{m}\right|_{0} \gamma^{2}$ at these fixed points are given by $-\alpha / \nu$ and $\alpha / \nu$, respectively.

Since $\alpha<0$ in the three-component case we are concerned with $(\alpha \simeq-0.12$ for $d=3$, according to Ref. 56), the infrared-stable fixed point is $(U, \gamma)=\left(u^{*}, 0\right)$. The results of Ref. 59 imply that the running coupling constant associated with $\gamma$ tends to zero as $m^{-\alpha / 2 \nu}$ in the limit $m \rightarrow 0$. Thus the energy density decouples asymptotically from the order parameter, so that the result (A5) applies.

We can introduce the renormalized transport coefficient $\lambda^{(\mathcal{E})}$ via $\lambda_{0}^{(\mathcal{E})}=m^{-2} Z_{\mathcal{E}} \lambda^{(\mathcal{E})}$, where $Z_{\mathcal{E}}$, the static renormalization factor of the energy density, takes the value 1 at the infrared-stable fixed point. The ratio of transport coefficients $\lambda / \lambda^{(\mathcal{E})}$ has the asymptotic scale dependence $\sim m^{\mathfrak{z}-\mathfrak{z} \varepsilon}$. If we substitute the values (71) and (A5) for $\mathfrak{z}$ and $\mathfrak{z} \mathcal{E}$, the exponent becomes $\mathfrak{z}-\mathfrak{z} \mathcal{E}=(d-2-\eta) / 2$. Since this is positive in three dimensions, the ratio approaches zero in the long-scale limit $m \rightarrow 0$. The upshot is that the critical dynamics of the order parameter remains unaffected by the coupling to the energy density, as claimed.

* Present address: Institut für Theoretische und Angewandte Physik, Universität Stuttgart, 70550 Stuttgart and Max-Planck-Institut für Metallforschung, Heisenbergstr. 1, 70569 Stuttgart, Federal Republic of Germany

1 M. E. Fisher, Rev. Mod. Phys. 46, 597 (1974); 70, 653 (1998).

2 M. E. Fisher, in Critical Phenomena, edited by F. J. W. Hahne (Springer-Verlag, Berlin, 1983), vol. 186 of Lecture Notes in Physics, pp. 1-139. 
3 B. I. Halperin and P. C. Hohenberg, Rev. Mod. Phys. 49, 435 (1977).

4 K. Binder, in Phase Transitions and Critical Phenomena, edited by C. Domb and J. L. Lebowitz (Academic, London, 1983), vol. 8, pp. 1-144.

5 H. W. Diehl, in Phase Transitions and Critical Phenomena, edited by C. Domb and J. L. Lebowitz (Academic Press, London, 1986), Vol. 10, pp. 75-267; Int. J. Mod. Phys. B 11, 3503 (1997), preprint cond-mat/9610143.

6 This happens, e.g., in the case of model $\mathrm{B}$ whose semi-infinite extensions $\mathrm{B}_{\mathrm{A}}$ and $\mathrm{B}_{\mathrm{B}}$, differing by the presence or absence of relevant nonconservative surface terms, represent distinct dynamic surface universality classes; see Refs. 7, 8, and 9 .

7 H. W. Diehl and H. K. Janssen, Phys. Rev. A 45, 7145 (1992).

8 H. W. Diehl, Phys. Rev. B 49, 2846 (1994).

9 F. Wichmann and H. W. Diehl, Z. Phys. B 97, 251 (1995).

10 S. Dietrich and H. W. Diehl, Z. Phys. B 51, 343 (1983), [Erratum 52, 171 (1983)].

11 D. Frank and V. Dohm, Phys. Rev. Lett. 62, 1864 (1989); Z. Phys. B 84, 443 (1991).

12 G. M. Xiong and C. D. Gong, Z. Phys. B 74, 379 (1989); J. Phys. Condens. Matter 1, 8673 (1989).

13 L. Mailänder, H. Dosch, J. Peisl, and R. L. Johnson, Phys. Rev. Lett. 64, 2527 (1990).

14 H. Dosch, Critical Phenomena at Surfaces and Interfaces, vol. 126 of Springer Tracts in Modern Physics (Springer, Berlin, 1992).

15 S. Krimmel, W. Donner, B. Nickel, H. Dosch, C. Sutter, and G. Grübel, Phys. Rev. Lett. 78, 3880 (1997).

16 For a detailed exposition of the theoretical background on surface X-ray and neutron scattering, see S. Dietrich and H. Wagner, Phys. Rev. Lett. 51, 1469 (1983); Z. Phys. B 56, 207 (1984); ibida 59, 35 (1985); S. Dietrich and A. Haase, Phys. Rep. 260, 1 (1995).

17 TESLA Technical Design Report, Part V, DESY, March 2001, http://tesla.desy.de/tdr/.

18 M. Krech, H. Karl, and H. W. Diehl, Physica A 297, 64 (2001), cond-mat/0102131.

19 S. Ma and G. F. Mazenko, Phys. Rev. B 11, 4077 (1975).

20 K. Kawasaki, Prog. Theor. Phys. 54, 1665 (1975).

21 H.-K. Janssen, Z. Phys. B 23, 377 (1976).

22 R. Bausch, H. K. Janssen, and H. Wagner, Z. Phys. B 24, 113 (1976).

23 V. Dohm, Solid State Comm. 20, 657 (1976). 
24 H. K. Janssen, in Dynamical Critical Phenomena and Related Topics, edited by C. P. Enz (Springer-Verlag, Berlin, 1979), pp. 25-47.

25 H. W. Diehl and M. Shpot, Phys. Rev. Lett. 73, 3431 (1994).

26 H. W. Diehl and M. Shpot, Nucl. Phys. B 528, 595 (1998), cond-mat/9804083.

27 S. Dietrich and H. W. Diehl, Z. Phys. B 43, 315 (1981).

28 D. M. McAvity and H. Osborn, Nucl. Phys. B 406, 655 (1993).

29 M. Krech, A. Bunker, and D. P. Landau, Comp. Phys. Commun. 111, 1 (1998); D. P. Landau, S.-H. Tsai, M. Krech, and A. Bunker, Int. J. Mod. Phys. 10, 1541 (1999); D. P. Landau, A. Bunker, H. G. Evertz, M. Krech, and S.-H. Tsai, Prog. Theor. Phys. Suppl. 138, 423 (2000).

30 D. P. Landau and M. Krech, J. Phys. Cond. Mat. 11, R179 (1999); M. Krech and D. P. Landau, Phys. Rev. B 60, 3375 (1999).

31 M. Krech, Phys. Rev. B 62, 6360 (2000), cond-mat/0006448, and references therein.

32 H. K. Janssen, in From Phase Transitions to Chaos, edited by G. Györgyi, I. Kondor, L. Sasvári, and T. Tel (World Scientific, Singapore, 1992), pp. 68-91.

33 C. de Dominicis, J. Phys. (Paris) Colloq. 37, C1 (1976).

34 P. C. Martin, E. D. Siggia, and A. Rose, Phys. Rev. A 8, 423 (1973).

35 H. Karl, Zum Einfluß von Oberflächen auf das dynamische kritische Verhalten von isotropen Heisenberg-Ferromagneten, Dissertation, U. Essen (Shaker-Verlag, Aachen, 2000).

36 D. J. Amit and L. Peliti, Ann. Phys. (USA) 140, 207 (1982).

37 The essence of this argument is nothing but a combination of the way in which the fluctuationdissipation theorem was exploited in Ref. 22 with the consequences implied by the minimal subtraction of poles. The discussion of the renormalization of insertions of the composite operator $\tilde{\phi} \times \phi$ given in this reference is restricted to single insertions. However, from our reasoning yielding the possible $\boldsymbol{K}$-dependent counterterms it is clear that the cumulants with arbitrarily many insertions, derived from the functional (60), must be uv finite as well.

38 In the bulk case, this vertex function, defined algebraically in the conventional way, simply turns out to be the operator inverse of $\left\langle\phi^{\alpha} \tilde{\phi}^{\beta}\right\rangle^{\text {cum }}$. In cases with boundaries as considered here, already the inverse of the free response propagator is uniquely defined only when considered as acting on a space of functions that satisfy the boundary conditions imposed on the free response propagator. The inverse then simply is given by $\delta^{2} / \delta \tilde{\phi}^{\alpha} \delta \phi^{\beta}$ of the Gaussian part of the action $\mathcal{J}$. The full $\Gamma_{\tilde{\phi}^{\alpha} \phi^{\beta}}$ may be considered to be defined graphically as being given by this zero-loop 
term minus the sum of all one-particle irreducible graphs with one external $\tilde{\phi}$ and one external $\phi$ leg.

39 This result may be gleaned from H. K. Janssen's lecture notes (unpublished) on his course on critical dynamics.

40 H. Wagner, Phys. Lett. 33A, 58 (1970).

41 The result (71) can also be derived for $T<T_{\mathrm{c}}$ via a simple scaling argument $13 \mathrm{1}$ According to hydrodynamics the long-wave-length spin waves must have a frequency $\omega_{q} \sim M q^{2}$, where $M$ is the (spontaneous) magnetization. Now $M \sim \xi^{-\beta / \nu}$ as $T \rightarrow T_{\mathrm{c}}-$. For $2<d<4$, where the hyperscaling law $\beta / \nu=(d-2+\eta) / 2$ holds, we thus have $\omega_{q} \sim \xi^{-(d+2+\eta) / 2}(q \xi)^{2}$. However, for $4<d<6$, hyperscaling is broken, $\beta / \nu$ takes its mean-field value 1 , one must take into account that $M \sim \xi^{-(d-2) / 2} / \sqrt{\bar{u}(\xi)}$, where $\bar{u}(\xi) \sim u \xi^{4-d}$ is the running $\phi^{4}$ coupling constant. The resulting scaling form $\omega_{q}=q^{1+d / 2} \Omega\left(q \xi, u \xi^{4-d}\right)$ reflects the dangerous character of the irrelevant variable $u$ and the concomitant necessity of including a second length ('thermodynamic length') besides $\xi$ in the scaling.

42 We do not consider the possibility that the $O(3)$ symmetry of the Hamiltonian is broken by surface terms such as a surface magnetic field or surface spin anisotropies. In the former case, a normale surface transition would be possible. Likewise, an easy-axis surface spin anisotropy could give rise to an anisotropic special transition 13 in three dimensions if the corresponding surface interaction constant is sufficiently enhanced.

43 H. W. Diehl and E. Eisenriegler, Phys. Rev. B 30, 300 (1984).

44 H. W. Diehl and S. Dietrich, Phys. Lett. 80A, 408 (1980).

45 H. W. Diehl and S. Dietrich, Z. Phys. B 42, 65 (1981), erratum: 43, 281 (1981).

46 K. Symanzik, Nucl. Phys. B 190, 1 (1981).

47 M. Krech, E. Eisenriegler, and S. Dietrich, Phys. Rev. E 52, 1345 (1995).

48 A definition of this renormalized surface enhancement $c$ can be found in 3.2 of Ref. 26, but will not be needed in the sequel.

49 Conservation of the order parameter implies that the zero-momentum contribution $\tilde{\phi}_{\boldsymbol{q}=\mathbf{0}}(t)$. $\dot{\phi}_{\boldsymbol{q}=\mathbf{0}}$ to the bulk action must retain its form upon renormalization, so that the trivial equation of motion $\dot{\boldsymbol{\phi}}_{\boldsymbol{q}=\mathbf{0}}=\partial_{t} \int d^{d} x \boldsymbol{\phi}=\mathbf{0}$ remains valid.

50 C. Bagnuls and C. Bervillier, Phys. Rev. B 24, 1226 (1981).

51 J. Zinn-Justin, Quantum Field Theory and Critical Phenomena, International series of mono- 
graphs on physics (Clarendon Press, Oxford, 1996), 3rd ed.

52 U. Wolff, Phys. Rev. Lett. 62, 361 (1989).

53 K. Chen, A. M. Ferrenberg, and D. P. Landau, Phys. Rev. B 48, 3249 (1993).

54 A. M. Ferrenberg, D. P. Landau, and Y. J. Wong, Phys. Rev. Lett. 69, 3382 (1993); L. N. Shchur and H. W. J. Blöte, Phys. Rev. E 55, R4905 (1997).

55 A. M. Ferrenberg and D. P. Landau, private communication.

56 R. Guida and J. Zinn-Justin, J. Phys. A 31, 8103 (1998).

57 The dimensionality expansion of its counterpart for $4 \leq d<6$, namely $\omega_{f} \equiv \beta_{f}^{\prime}\left(0, f^{*}\right)$, is known to first order: According to Ref. 22, it reads $\omega_{f}=6-d+O\left[(6-d)^{2}\right]$.

58 If one were naive, one might expect that the analog $\left(d+2-\eta_{\|}\right) / 2$ of the dynamic exponent $\mathfrak{z}=(d+2-\eta) / 2$ might play the role of the latter in surface quantities. However, this is not the case.

59 E. Brézin and C. de Dominicis, Phys. Rev. B 12, 4954 (1975). 